\title{
Synaptic Functions in Rat Sympathetic Neurons in Microcultures. II. Adrenergic/Cholinergic Dual Status and Plasticity
}

\author{
D. D. Potter, S. C. Landis, S. G. Matsumoto, and E. J. Furshpan \\ Department of Neurobiology, Harvard Medical School, Boston, Massachusetts 02115
}

This is the second in a series of papers that describes the use of a sensitive microculture procedure to investigate the transmitter status of sympathetic neurons. Cultured immature principal neurons, dissociated from the superior cervical ganglia of newborn rats, are known to be plastic with respect to transmitter status; under certain culture conditions, populations of neurons that display (at least) adrenergic properties at the outset can be induced to display a variety of cholinergic properties, including the formation of functional neuron-neuron cholinergic synapses, as adrenergic properties decline. With the microculture procedure described in the preceding paper (Furshpan et al., 1986a), we have examined the transmitter status of individual neonatederived neurons during this transition. Many such neurons secreted both norepinephrine and $\mathrm{ACh}$ (adrenergic/cholinergic dual function); examination of such neurons with the EM revealed a mixed population of synaptic vesicles. Direct evidence for a transition via this dual status was obtained by serial physiological assays of 14 neurons. The neonate-derived neurons were markedly heterogeneous in the rate of change of transmitter status.

Principal neurons derived from adult superior cervical ganglia also displayed dual status, but the incidence was lower than in neonate-derived neurons cultured for similar periods. In preliminary serial assays of adult-derived neurons, many of the neurons did not acquire detectable cholinergic function, but in two cases evidence consistent with plasticity was obtained.

While it is known that several types of neurons will form functional junctions in the presence of agents that block electrical activity, sympathetic principal neurons have apparently not been tested. In microculture, neuron-neuron synapses and junctions with cardiac myocytes were formed by sympathetic neurons grown chronically in the presence of blocking concentrations of TTX and hexamethonium.

The experiments described in this paper were a direct extension of earlier work on adrenergic and cholinergic properties displayed by immature sympathetic principal neurons developing in "mass" cultures (several thousand neurons per dish). This

Received Dec. 21, 1984; revised Sept. 18, 1985; accepted Sept. 23, 1985.

Dinah Sah participated in some of the experiments described in this paper. Expert assistance was provided by Robert Bosler, Wendy Brooks, Delores Cox, Karen Fischer, Joseph Gagliardi, Ibrahim Houri, James LaFratta, Michael LaFratta, Kathleen Lamb, Doreen McDowell, Claudia Miles, Geraldine Spencer, Shirley Wilson, and Vivienne Yee. $\mathrm{A}^{60} \mathrm{Co}$ source was kindly made available by Drs. J. Epstein and J. D. Little. Drs. Linda Chun, Allison Doupe, Keiko Fukada, Eve Wolinsky, and Geraldine Spencer provided conditioned medium or partially purified $\mathrm{CM}$ factor. We are indebted to many colleagues for helpful discussion, especially Drs. Linda Chun, Peter MacLeish, Paul O'Lague, and Paul Patterson. This work was supported by NIH Grants NS11576, NS03273, and NS02253, a Grant-In-Aid from the American Heart Association (78-964), and an American Heart Association Established Investigator Award to S.C.L.

Correspondence should be addressed to Professor David Potter, Department of Neurobiology, Harvard Medical School, 25 Shattuck Street, Boston, MA 02115. Copyright (C) 1986 Socicty for Neuroscicnce $0270-6474 / 86 / 041080-19 \$ 02.00 / 0$ earlier work provides a setting for the unconventional adrenergic/cholinergic dual status reported here; the points of special relevance to this paper are described below (for reviews, see Bunge et al., 1978; O'Lague et al., 1978b, c; Patterson, 1978).

During the first week in mass cultures, a very high proportion, perhaps all, of the neurons derived from the superior cervical ganglion of the newborn rat are at least adrenergic, for collectively they synthesize and store dopamine and norepinephrine (NE; Mains and Patterson, 1973), and at least a majority of varicosities and terminals take up and store catecholamines in small granular vesicles (SGV; Johnson ct al., 1976) or contain vesicles with endogenous granules after permanganate fixation (Landis, 1978, 1980). If these neurons are cocultured with cardiac cells or fed medium previously conditioned by such cells (conditioned medium, CM), cholinergic properties appear over a period of several weeks (MacLeish, 1977; Patterson and Chun, 1974,1977 a). This induction is graded in the sense that the higher the proportion of CM in the medium, the greater the synthesis and storage of $\mathrm{ACh}$ at a particular culture age and the higher the incidence of synapses of cholinergic function and fine structure (Landis et al., 1976; see also Landis, 1980). In culture conditions that strongly promote this transition, synthesis and storage of NE are progressively suppressed as synthesis of $\mathrm{ACh}$ rises (Patterson and Chun, 1977b; Wolinsky and Patterson, 1983), the majority of the neurons form functional cholinergic synapses on neighboring neurons, and the fine structure of neuron-neuron synapses becomes increasingly cholinergic (Johnson et al., 1976, 1980; Landis, 1980; MacLeish, 1977; O'Lague et al., 1976, 1978c). Strong indirect evidence was obtained that this change in transmitter state in the mass cultures arises as a transition in a population of plastic neonatal neurons, not as a result of selection between two populations, each capable of expressing only one of these states (Johnson et al., 1980; Landis, 1980; Patterson and Chun, 1977a). The transition is slowed or blocked by raising the concentration of $\mathrm{K}^{+}$in the medium to $20 \mathrm{~mm}$ ["high-K+" medium (Landis, 1980; Walicke et al., 1977)].

These findings on mass cultures raised questions about the behavior of individual cultured neurons during the transition: Does a neuron lose adrenergic function before incorporating cholinergic properties (transition via a null state) or are cholinergic properties added in a graded way as adrenergic properties are phased out (transition via adrenergic/cholinergic dual status)? Are the neurons synchronous in their transitions, i.e., does the slow time course of the transition in mass cultures reflect the time course in a single neuron? A major motivation for developing the microculture procedure was to investigate these questions in individual neurons. Preliminary reports of some of these findings were made by Furshpan et al. (1976, 1982), Landis (1976), and Potter et al. (1980, 1981b, c, 1983).

\section{Materials and Methods}

The methods for establishing and maintaining microcultures of neonateor adult-derived sympathetic principal neurons and cardiac cells were identical to these described in the preceding paper, Furshpan et al. 


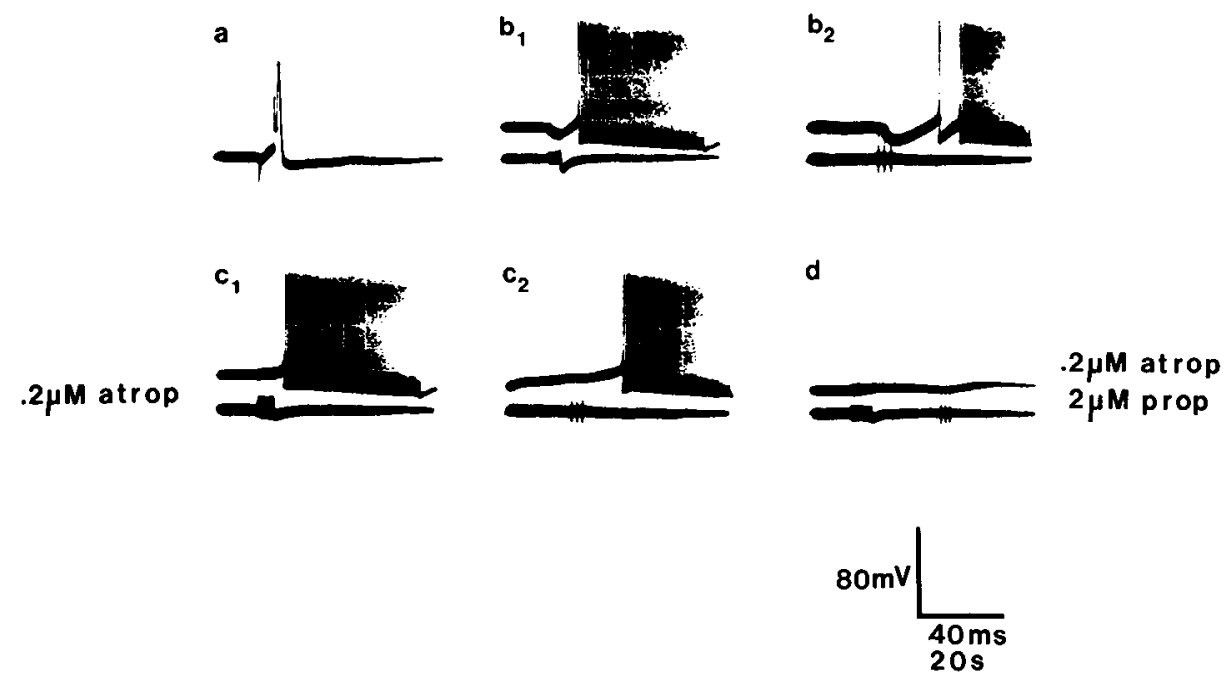

Figure 1. Adrenergic/cholinergic dual function mimicked by "puffs" of a mixture of ACh and NE. The solitary neonate-derived neuron was grown for $21 \mathrm{~d}$ in high- $\mathrm{K}^{+}$medium. An impulse in this neuron, as in some others grown in this medium, was not followed by an autaptic EPSP (a). In $b_{1}$, a train of neuronal impulses (deflection of lower trace; $50 \mathrm{~Hz}$ for about $2.5 \mathrm{sec}$ ) evoked a hyperpolarization of the myocytes (upper trace) followed by a train of myocyte impulses that lasted about $30 \mathrm{sec}$. This dual effect was mimicked by 3 puffs of $5 \mu \mathrm{M}$ ACh and $100 \mu \mathrm{M}$ NE $\left(b_{2}\right)$; each puff, 100 msec duration, is marked by a deflection of the lower trace; the train of myocyte impulses lasted for another 13 sec on the next sweep (not shown). Addition of $0.2 \mu \mathrm{M}$ atropine sulfate blocked the neuronally evoked hyperpolarization $\left(c_{1}\right)$ and sharply reduced that produced by the puffs $\left(c_{2}\right)$. The remaining excitatory effects were blocked by further addition of $2 \mu \mathrm{M}$ propranolol $(d)$; note that both the neuronal stimulation and the puffs were applied during the trace of $d$. Scales: $80 \mathrm{mV}$ for $a$ and myocyte traces in $b_{1}-d ; 40$ msec for $a, 20$ sec for all other traces.

(1986a), as were the methods for recording from the cells, identifying the transmitters physiologically, and subsequently processing the cultures for electron microscopy.

When serial assays were planned, precautions were taken to reduce the risk of contamination of the culture dish with microorganisms during the recording session; because the media contained penicillin and streptomycin, contamination by molds and yeasts was the primary concern. A Lucite hood on the microscope stage diminished air currents over the open culture dish during a recording session. The microscope stage, the stainless steel insert, the perfusion system, and the agar bridge that served as a bath electrode (see O'Lague et al., 1978a) were autoclaved or sterilized with $70 \%$ ethyl alcohol before the culture dish was placed on the stage. Drug solutions were sterilized by filtration. After the recording session, the culture was rinsed several times with sterile medium and returned to the incubator. Visible contamination of the dish with microorganisms ended the experiments; about four out of every five assays were terminated prematurely in this way. In a few cases, the neuron was evidently irreversibly damaged by impalement in the first assay, as it died after the culture was returned to the incubator.

In some serial assays of neonate-derived neurons, the culture was fed at the outset with high- $\mathrm{K}^{+}$medium (composition given in Furshpan et al., 1986a) to reduce the responsiveness of the neurons to the "cholinergic" influence of the cardiac cells on which the neurons were grown (Walicke et al., 1977) and of the adult rat serum present in the medium (Wolinsky and Patterson, 1985), and to increase thereby the probability that at first assay the neuron would exhibit purely adrenergic function. After the first assay, in some cases, the culture was fed CM (medium previously conditioned by cardiac cells and mixed, often 1:1, with normal growth medium) in an attempt to hasten the adrenergic-to-cholinergic transition; in some cases, partially purified CM factor was added to the growth medium.

Several figures in this paper show a purinergic effect exerted by the neurons on the myocytes; this effect is the subject of the next paper in this series (Furshpan et al., 1986b) and is described in detail there. To block the purinergic effect, 8-phenyltheophylline (8-PT; Calbiochem) was used. This agent has limited solubility in the perfusion medium. To achieve a blocking concentration of $1-3 \mu \mathrm{M}$, a suspension (about 10 $\mu \mathrm{mol} /$ liter) was stirred for 1-3 $\mathrm{hr}$ and filtered to remove undissolved 8PT. Alternatively, the agent was dissolved at a concentration of $3 \mu \mathrm{M}$ in dimethylsulfoxide and diluted 1000 -fold with perfusion fluid, which was then filtered. Perfusion with this concentration of dimethylsulfoxide alone had no consistent effect on the neurons or myocytes. In either case, there was uncertainty about the final concentration of 8-PT, which was therefore called "nominally $3 \mu \mathrm{M}$." The sources of the other drugs used are given in Furshpan et al. (1986a). Figure 8 shows a nonadrenergic excitatory (NAE) effect on the myocytes, described in detail elsewhere (Matsumoto et al., in press; D. Sah and S. G. Matsumoto, unpublished observations).

\section{Results}

In the preceding paper (Furshpan et al., 1986a), it was reported that some sympathetic neurons microcultured on cardiac myocytes displayed the classical transmitter states: The neurons were purely adrenergic or cholinergic within the sensitivity of the assays. We report here that a larger proportion of microcultured neurons, assayed once at various times after the onset of culture (singly assayed neurons) or serially assayed at two or more times, displayed adrenergic/cholinergic dual function.

\section{Adrenergic/cholinergic dual status in singly assayed neonate-derived neurons}

Many solitary neurons produced a dual effect on the cocultured cardiac myocytes, a hyperpolarization or reduction in the frequency of spontaneous beating that was sensitive to block by atropine, followed by a depolarization or an increase in the frequency of beating that was sensitive to adrenergic blockers (propranolol, or atenolol plus phentolamine). An example of this dual status is shown in Figure 1. In this microculture, only one neuronal cell body was visible with phase microscopy; in some other examples described below, the solitary status of the neuron was confirmed by electron microscopy of semiserial thin sections. The microculture was grown in high- $\mathrm{K}^{+}$medium to delay onset of cholinergic function (Walicke et al., 1977). In Figure 1, the effects on the myocytes produced by trains of neuronal impulses $\left(b_{1}, c_{1}, d\right)$ are compared with the effects of "puffs" of a mixture of ACh and NE from a micropipette positioned close to the myocytes in $b_{2}, c_{2}$, and $d$. The hyperpolarizations produced by neuronal stimulation and by the puffs of authentic $\mathrm{ACh}$ were blocked by atropine sulfate $\left(c_{1}, c_{2}\right)$. When propranolol, a $\beta$-adrenergic blocker, was added to atropine in the perfusion fluid, neuronal activity had no detectable effect on the myocytes, and the puffs of ACh and NE now produced 
only a slight hyperpolarization followed by a slight depolarization $(d)$. When the two blockers were washed out, the adrenergic and cholinergic effects were restored (not shown).

The sensitivity of the neuronally evoked hyperpolarization to atropine, the sensitivity of the excitatory effect to propranolol, and the parallel action and sensitivity to antagonists of authentic $\mathrm{ACh}$ and $\mathrm{NE}$ leave no doubt that this solitary neuron secreted both $\mathrm{ACh}$ and NE (cf. evidence for cholinergic and adrenergic functions in Furshpan et al., 1986a). In our preliminary report of dual status (Furshpan et al., 1976; Landis, 1976), dual secretion and dual fine structure (illustrated below) were not taken as an unequivocal demonstration of dual synthesis by a solitary neuron, as it was imaginable that NE, synthesized by adrenergic neurons elsewhere in the array of microcultures, was released into the culture medium, taken up, and secreted by neurons that were themselves capable of synthesizing only ACh. However, we have now encountered several dual-function neurons that were the only neuron in the dish, and we see no reason to doubt that microcultured neurons of dual status synthesized both transmitters.

In cases of dual function like that of Figure 1, where both effects were pronounced, the cholinergic inhibition of the myocytes always preceded the adrenergic excitation. It is plausible that these sequential effects were produced by simultaneous secretion of the two transmitters inasmuch as simultaneous application of the two transmitters in puffs produced a similar effect, and the latency of intense cholinergic effects was shorter than the latency of intense adrenergic effects (Furshpan et al., 1986a). As described below, the terminals of dual-function neurons had a mixed fine structure.

Adrenergic/cholinergic function was common in the microcultures (in several samples, $45-66 \%$ of neonate-derived neurons exhibited both functions; see below). The existence of cultured neurons that secreted both transmitters is consistent with the idea that the neonatal superior cervical ganglion of the rat contains principal neurons capable of a transition from adrenergic to cholinergic status and is obviously inconsistent with the idea that all of the neurons in the neonatal ganglion are already committed to only one status. Additional evidence consistent with such a transition is the graded intensity of the two effects exhibited by dual-function neurons, as described in the next section.

\section{Singly assayed dual-function neurons differed in the relative strength of their adrenergic and cholinergic effects on the myocytes}

A conspicuous feature of the neurons that secreted both $\mathrm{ACh}$ and NE was their variation from predominantly adrenergic to predominantly cholinergic. Neurons that were apparently purely adrenergic or cholinergic in the preceding paper (Furshpan et al., 1986a) may be considered the extremes of this spectrum. In the neuron of Figure 1, adrenergic function was relatively strong; a high-frequency train of impulses $\left(b_{1}\right)$ gave rise to rather slow cholinergic hyperpolarization of the myocytes (compare with Fig. 8) that was followed by a prolonged intense adrenergic excitation (high-frequency beating of the myocytes; note also the absence of cholinergic autaptic EPSPs in Fig. 1a). Many neurons produced adrenergic and cholinergic effects on the myocytes that appeared more balanced than that of Figure 1; an example is neuron $N_{1}$ of Figure 2 . In contrast, the relative strength of the cholinergic effect of neuron $\mathrm{N}_{2}$ in Figure 2 was sufficient to mask the adrenergic effect in control solution $\left(b_{22}\right.$ and $\left.e_{2}\right)$. A case in which a powerfully cholinergic neuron produced a possibly adrenergic effect so weak as to be at the limit of detection of the assay, shown in Figure 8 (neuron $N_{1}$ ), is described below.

A factor that we did not systematically investigate or control and that might have contributed to this variation was differences from microculture to microculture in the sensitivity of the my- ocytes to the transmitters. However, in all tests with "puffed" $\mathrm{ACh}$ and NE the myocytes were found to be responsive to both agents (Furshpan et al., 1986a). In addition, observations on two-neuron microcultures sometimes provided an internal control for the responsiveness of the myocytes. In the microculture of Figure 8, described in detail below, one neuron was strongly cholinergic and moderately purinergic and produced a NAE effect; it was possibly also very weakly adrenergic (purinergic and NAE effects are described in detail in Furshpan et al., 1986b, and Matsumoto et al., in press). The other neuron was apparently purely (and effectively) adrenergic. Thus, the effects evoked by each neuron confirmed the responsiveness of the common pool of coupled myocytes to the transmitter function(s) expressed weakly or undetectably by the other neuron. Three other factors that varied from microculture to microculture-the frequency and duration of neuronal stimulation, the density of neuronal varicosities, and the level of myocyte membrane potential or spontaneous activity-would plausibly have affected the apparent intensity of the two transmitter actions at least roughly in parallel; this is supported by many cases in which both effects were weak or both were strong. From these considerations, we tentatively conclude that the variation in the relative strengths of the adrenergic and cholinergic effects, from microculture to microculture, primarily reflected variations in transmitter status associated with the transition.

Neurons $\mathrm{N}_{1}$ and $\mathrm{N}_{2}$ of Figure 2 apparently differed in the relative strengths of their adrenergic and cholinergic functions, although they were plated onto adjacent microcultures on the same day from the same suspension of dissociated neonatal neurons and shared the same medium. The possible relevance of such heterogeneity in status to the adrenergic-to-cholinergic transition is discussed in a later section.

\section{Fine structure associated with adrenergic/cholinergic dual function}

Physiologically characterized neurons were prepared for electron microscopy by procedures described in Furshpan et al. (1986a). To ensure that the assayed neuron was relocated for ultrastructural analysis, the address of the microculture in the $5 \times 5$ array was noted and identifying features were sketched. After permanganate fixation, the junctions and varicosities of dual-function neurons were found to contain a mixture of SGV, plausibly storage sites for $\mathrm{NE}$, and small clear vesicles (SCV), many of which plausibly stored ACh. Morcover, the relative numbers of the two vesicle types roughly corresponded to the relative intensities of adrenergic and cholinergic functions determined electrophysiologically for the same neuron. These points are illustrated in Figure 3, which compares the terminals of neurons $N_{1}$ and $N_{2}$ of Figure 2. The proportion of $S G V$ was much higher in the junctions of $\mathrm{N}_{1}$ (Fig. 3, $a$ and $b$ ) than in $\mathrm{N}_{2}$ (Fig. $3 c$ ), consistent with the finding that adrenergic function was relatively stronger in $\mathrm{N}_{1}$ than in $\mathrm{N}_{2}$. To obtain further information about these vesicle populations, synapses and varicosities containing a substantial number of vesicles were photographed, and the numbers of clear and granular vesicles were counted. The histograms of Figure $3, d$ and $e$, show the proportions of junctions that contained various percentages of SGV. There was almost no overlap in the two distributions. The mean proportions of SGV were $25 \%$ for $\mathrm{N}_{1}$ and $3 \%$ for $\mathrm{N}_{2}$. Eight other solitary neurons in which such vesicle counts were made also showed parallel variations in the relative strength of adrenergic function and the percentage of SGV.

A point of interest about the histograms of Figure 3, $d$ and $e$, is that while the synapses and varicosities of a given neuron were not identical in their proportions of SGV, they nevertheless appeared to form a single population rather than two distinct populations, one apparently cholinergic and the other apparently adrenergic. In a neuron like $\mathrm{N}_{2}$ in which the incidence of 

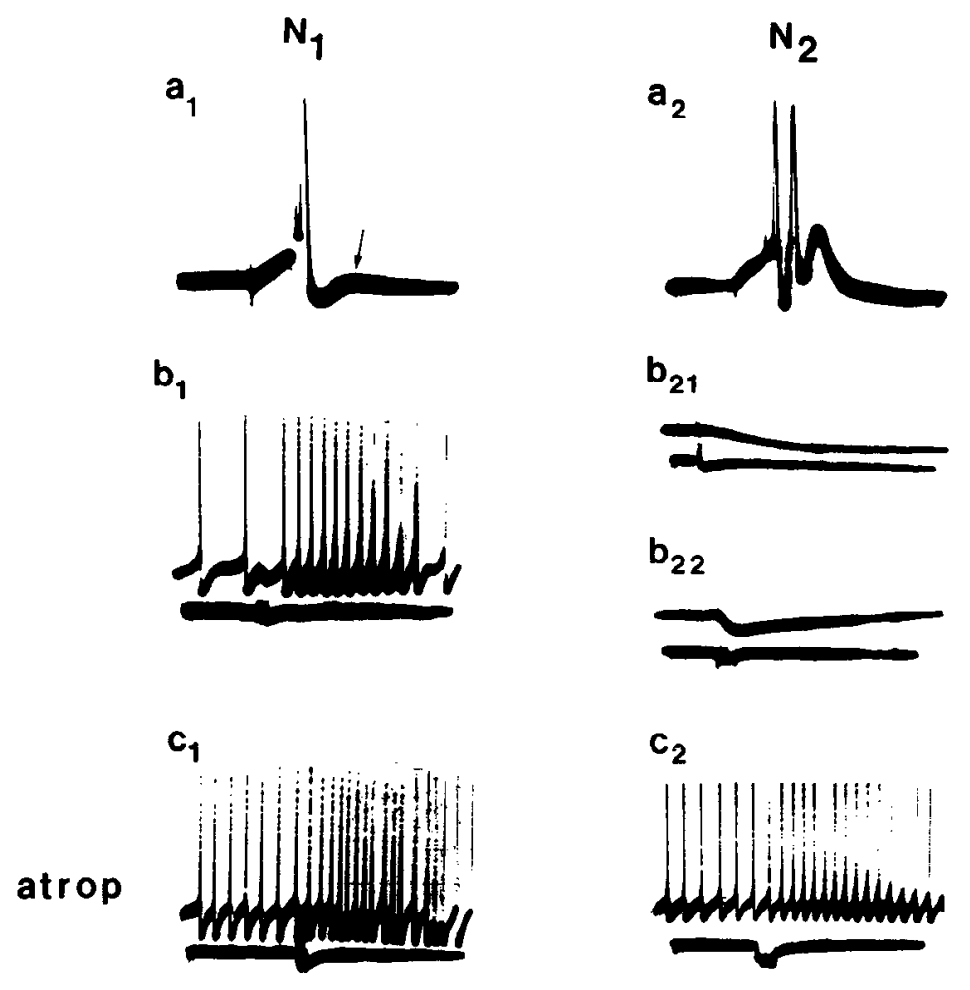

$\mathrm{c}_{2}$

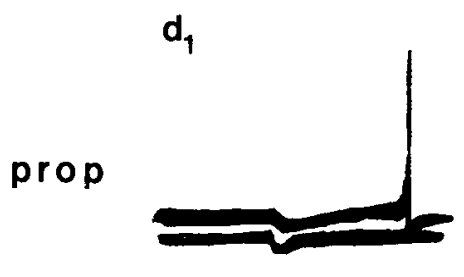

$\mathbf{d}_{2}$
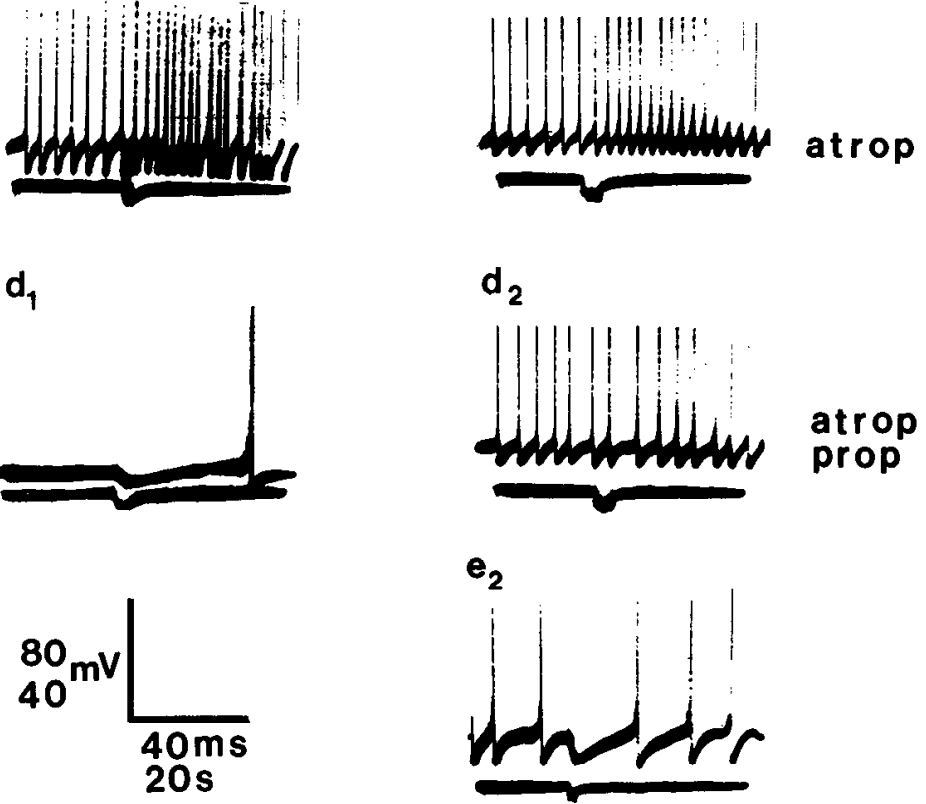

Figure 2. Adrenergic and cholinergic functions of different relative strengths in two solitary, neonate-derived neurons from adjacent microcultures in the same dish. The neurons were grown in high- $\mathrm{K}^{+}$medium for $11 \mathrm{~d}$ and then in $50 \% \mathrm{CM}$ until day 24 . The fine structure of junctions of these neurons is illustrated in Figure 3. Neuron $N_{1}\left(a_{1}-d_{1}\right)$ conspicuously displayed both transmitter states and in a more balanced way than the neuron of Figure 1 or the other neuron $\left(\mathrm{N}_{2}\right)$ of this figure. An impulse in $\mathrm{N}_{1}\left(a_{1}\right)$ was followed by a small cholinergic autaptic EPSP (arrow). In $b_{1}$, a train of neuronal impulses $(50 \mathrm{~Hz}$ for about $2 \mathrm{sec}$ ) evoked a small hyperpolarization of the myocytes, followed by an increased frequency of myocyte activity. The excitatory adrenergic effect on the myocytes is seen in isolation in $c_{1}$ in the presence of $0.1 \mu \mathrm{M}$ atropine sulfate (neuronal impulses at $20 \mathrm{~Hz}$ for about $2 \mathrm{sec}$ ). (Note: Addition of atropine often initiated, or increased the frequency of, beating of the myocytes.) The inhibitory cholinergic effect is seen in isolation in $d_{1}$ in the presence of $1 \mu \mathrm{M}$ propranolol; the myocyte impulse may have been spontaneous (neuronal impulses at $20 \mathrm{~Hz}$ for about $3 \mathrm{sec}$ ). In contrast, neuron $\mathrm{N}_{2}\left(a_{2}-e_{2}\right)$ was relatively more cholinergic, so that in normal perfusion fluid no adrenergic effect was visible. $\mathrm{N}_{2}$ had a pronounced autaptic effect $\left(a_{2}\right)$. A single stimulus ( 2 neuronal impulses as in $a_{2}$ ) hyperpolarized the myocytes $\left(b_{21}\right)$ with a short latency of about $40 \mathrm{msec}$, an effect characteristic of strongly cholinergic neurons (see Furshpan et al., 1986a). A train of neuronal impulses $\left(b_{22}\right.$; $10 \mathrm{~Hz}$ for about $3 \mathrm{sec}$ ) seemed only to hyperpolarize the myocytes. However, in the presence of $0.12 \mu \mathrm{M}$ atropine, a weak excitatory effect was unmasked ( $c_{2}$; stimulation at $20 \mathrm{~Hz}$ for about $3 \mathrm{sec})$; when this was blocked by further addition of $1 \mu \mathrm{M}$ propranolol, neuronal activity was ineffective $\left(d_{2}\right.$; stimulation at $20 \mathrm{~Hz}$ for about $3 \mathrm{sec}$ ). Removal of the blocking agents $\left(e_{2}\right)$ restored the apparently purely-inhibitory effect (stimulation at $20 \mathrm{~Hz}$ for about 1.5 sec). Vertical scale: $40 \mathrm{mV}$ for $a_{1}, a_{2}$ and the myocyte traces in $b_{1}, c_{1}, d_{1}, b_{21}, b_{22}, e_{2} ; 80 \mathrm{mV}$ for $c_{2}$ and $d_{2}$. Horizontal scale: 40 msec for $a_{1}$ and $a_{2}$; $400 \mathrm{msec}$ for $b_{21} ; 20 \mathrm{sec}$ for all other traces.

SGV was quite low, some varicosities in a thin section occasionally contained no SGV, while other varicosities contained a few SGV. In two such neurons, several varicosities were followed in serial sections, and varicosities that appeared to lack SGV in one section contained SGV in adjacent sections. All the solitary neurons in which vesicle counts were made showed only a single population of terminals. The implication is that cho- linergic and adrenergic properties coexist within each terminal, and that the synapses and varicosities are individually dual in status like the neuron as a whole. Moreover, in all microcultures examined, no pronounced difference was seen between the vesicle populations in synapses on the cell body and in varicosities near the myocytes.

At the beginning of this paper the question was raised whether 

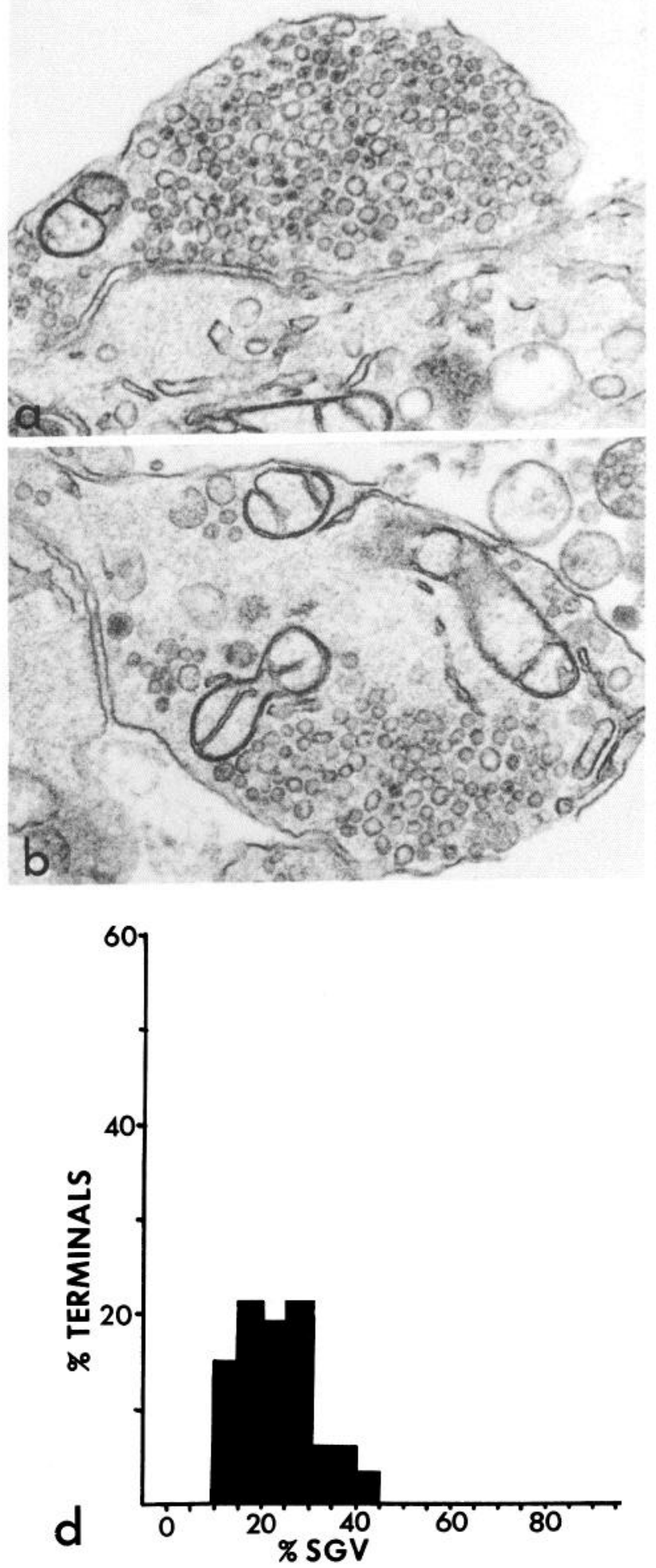
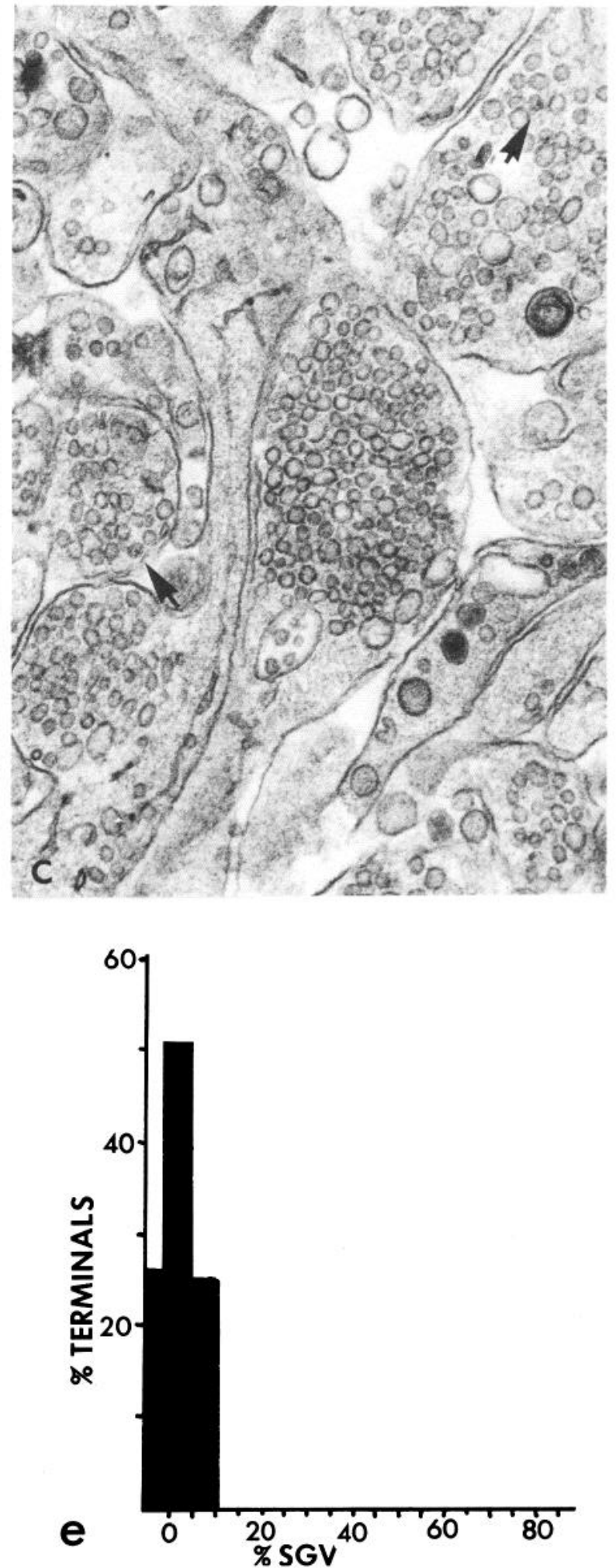

Figure 3. Junctional fine structure of the two solitary neonate-derived neurons of Figure 2 that differed in the relative strengths of their adrenergic and cholinergic functions. After the physiological assay shown in Figure 2, the two microcultures were fixed in $4 \%$ potassium permanganate. Two autapses of $\mathrm{N}_{1}$ are shown in $a$ and $b$; varicosities of $\mathrm{N}_{2}$ are shown in $c$. The proportion of $\mathrm{SGV}$ in $\mathrm{N}_{1}(a, b)$ was clearly higher than in $\mathrm{N}_{2}(c$; arrows indicate SGV), just as adrenergic function was relatively stronger in $\mathrm{N}_{1}$. In each case, counts were made of the percentage of SGV in junctions in which a substantial number of small synaptic vesicles were present: 32 junctions of $\mathrm{N}_{1}$ and 44 of $\mathrm{N}_{2}$. These measurements are plotted against the percentage of terminals of each kind (bin widths $5 \%$ ), in $d$ for $\mathrm{N}_{1}$ and in $e$ for $\mathrm{N}_{2}$ (the first bin in $e$ represents sections of varicosities that lacked SGV): Each neuron appeared to have only one type of terminal with a somewhat variable mixed fine structure, rather than two types of terminals (i.e., one type with a high proportion of SGV and the other with SCV only). Magnification, $\times 40,500$ for $a-c$. 
the cultured neurons undergo the adrenergic-to-cholinergic transition via dual status or null status. The presence of dual status in many singly assayed neurons obviously suggests that the transition can occur via this route; direct evidence comes from the serial assays described in the next section. According to this interpretation, neurons with different relative strengths of adrenergic and cholinergic function are at different stages of the transition. Differences in the rate of transition are suggested by the observations in Figures 2 and 3. While it appears that the whole neuron changes gradually in status (rather than adding new purely cholinergic endings among existing adrenergic endings), the observations in Figure 3 do not rule out some asynchrony in the transition among the endings of a neuron.

\section{Serial assays of microcultured neonate-derived neurons}

The ease with which a particular neuron could be identified in the culture dish permitted repetition of the physiological assay so that the transmitter status of that neuron could be followed over time. The principal motivations for performing the serial assays were to provide direct evidence for the adrenergic-tocholinergic transition in neonate-derived neurons, for which much indirect evidence had been previously obtained in mass cultures, and to investigate the rapidity of the transition in individual neurons. Figure 4 summarizes 27 experiments, on neonate-derived neurons, in which two or three serial assays were performed and the status of the neuron at each assay was reasonably clear. All but four of the experiments were performed before the purinergic and NAE functions were characterized, and some of the serial assays of transmitter status would not have revealed minor purinergic or NAE effects (the records from the myocytes were at low amplification, or the trains of neuronal impulses were brief or at a low frequency). In 13 of the 27 cases (Fig. $4 a$ ), there was no clear change in the relative intensities of the adrenergic and cholinergic effects on the myocytes, although in 3 of these cases an autaptic effect or synaptic effect on a second neuron in the same microculture became stronger. All exhibited adrenergic/cholinergic dual function except for the neuron assayed on days 12 and 19, which had no detectable effect on itself or the myocytes at either assay (null-null); this neuron had resting and action potentials similar to those of other serially assayed neurons. The lack of change in these 13 neurons is considered further in the Discussion.

The remaining 14 of the 27 neurons of Figure 4 underwent a change in status in the indicated intervals. The four ncurons in $b$ changed from apparently purely adrenergic to adrenergic/cholinergic status; the third neuron was assayed three times and had not detectably changed at the second assay after $6 \mathrm{~d}$ (like eight of the neurons in $a$ that were re-assayed after a similarly short interval). The five ncurons of $c$ changed from dual status to a relatively more cholinergic dual status. The cholinergic effect on the myocytes was larger on the second assay, and the adrenergic effect was about the same or smaller. The four neurons of $d$ changed from dual status to apparently purely cholinergic; the first of these neurons was grown in high- $\mathrm{K}^{+}$medium for the first $14 \mathrm{~d}$ and then in 50\% CM until the first assay on day 25 . One neuron $(e)$ changed from apparently purely adrenergic to dual status and then to apparently purely cholinergic, the full transition. These interpretations of the 14 cases rest on the assumption that the changes occurred in the transmitters secreted by the neurons and not in the sensitivity of the myocytes to ACh and NE, an assumption based on the fact that in all cases in which ACh and NE were puffed into microcultures, the myocytes responded strongly to both agents, as reported above (cf. Figs. 1 and 6). These interpretations are strengthened by the correlation between function and fine structure and are consistent with the earlier, less direct evidence from mass cultures for an adrenergic-to-cholinergic transition induced by cardiac cells (evidence reviewed in the introduction). It should be noted that

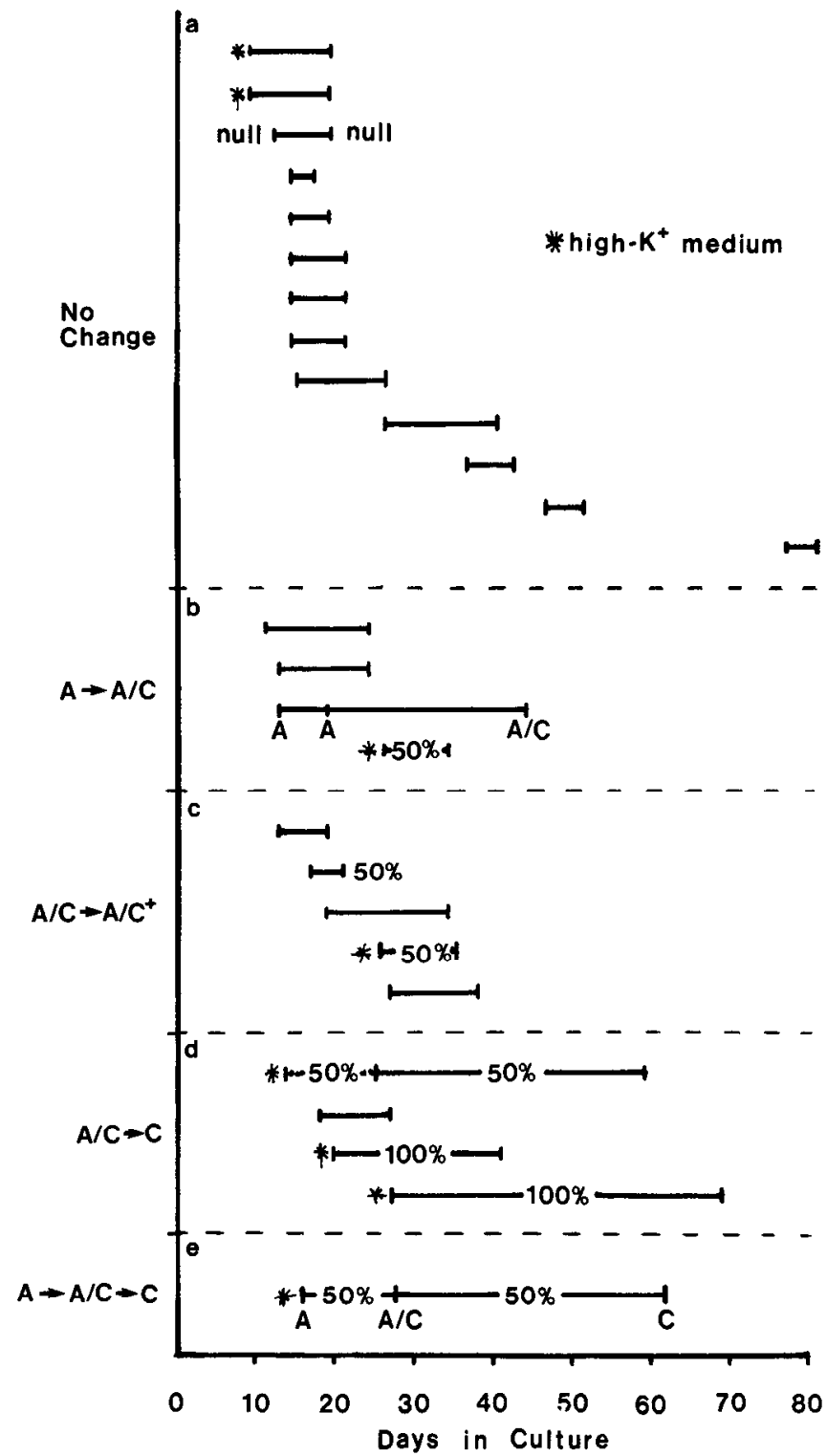

Figure 4. Summary of serial assays of 27 neonate-derived neurons. The age of the culture in days is shown on the horizontal axis. Each bar represents a serially assayed neuron; the left end of the bar indicates the age at first assay and the right end the age at the last assay. An asterisk indicates that the culture was grown in high- $\mathrm{K}^{+}$medium before the first assay; cultures grown after the first assay in 50 or $100 \% \mathrm{CM}$ (to promote cholinergic function) are so indicated. The assay schedules are given in five groupings: $a$, Thirteen neurons whose transmitter status did not change detectably between assays (No Change); one of these neurons had no detectable effect on itself or the myocytes at either assay (null-null). $b$, Four neurons that were apparently purely adrenergic at the first assay and dual in status at the last assay $(A \rightarrow A / C)$. $c$, Five neurons that changed from adrenergic/cholinergic status to a relativelymore-cholinergic status $\left(A / C \rightarrow A / C^{+}\right) . d$, Four neurons that changed from dual status to apparently purely cholinergic $(A / C \rightarrow C)$. $e$, One neuron that was apparently purely adrenergic at first assay, dual in status at second assay, and apparently purely cholinergic at third assay $(A \rightarrow$ $A / C \rightarrow C)$.

all of the transitions of Figure $4, b-e$, were in the relatively more cholinergic direction.

One of the partial transitions (A/C $\rightarrow \mathrm{C}$; neuron 4 in Fig. $4 d$ ) is illustrated in Figure 5, and the apparently complete transition of Figure $4 e$ is illustrated in Figure 6. At the first assay, the neuron of Figure 5 was cholinergic, as it evoked autaptic EPSPs $\left(a_{1}\right)$ and an atropine-sensitive hyperpolarization of the myocytes 


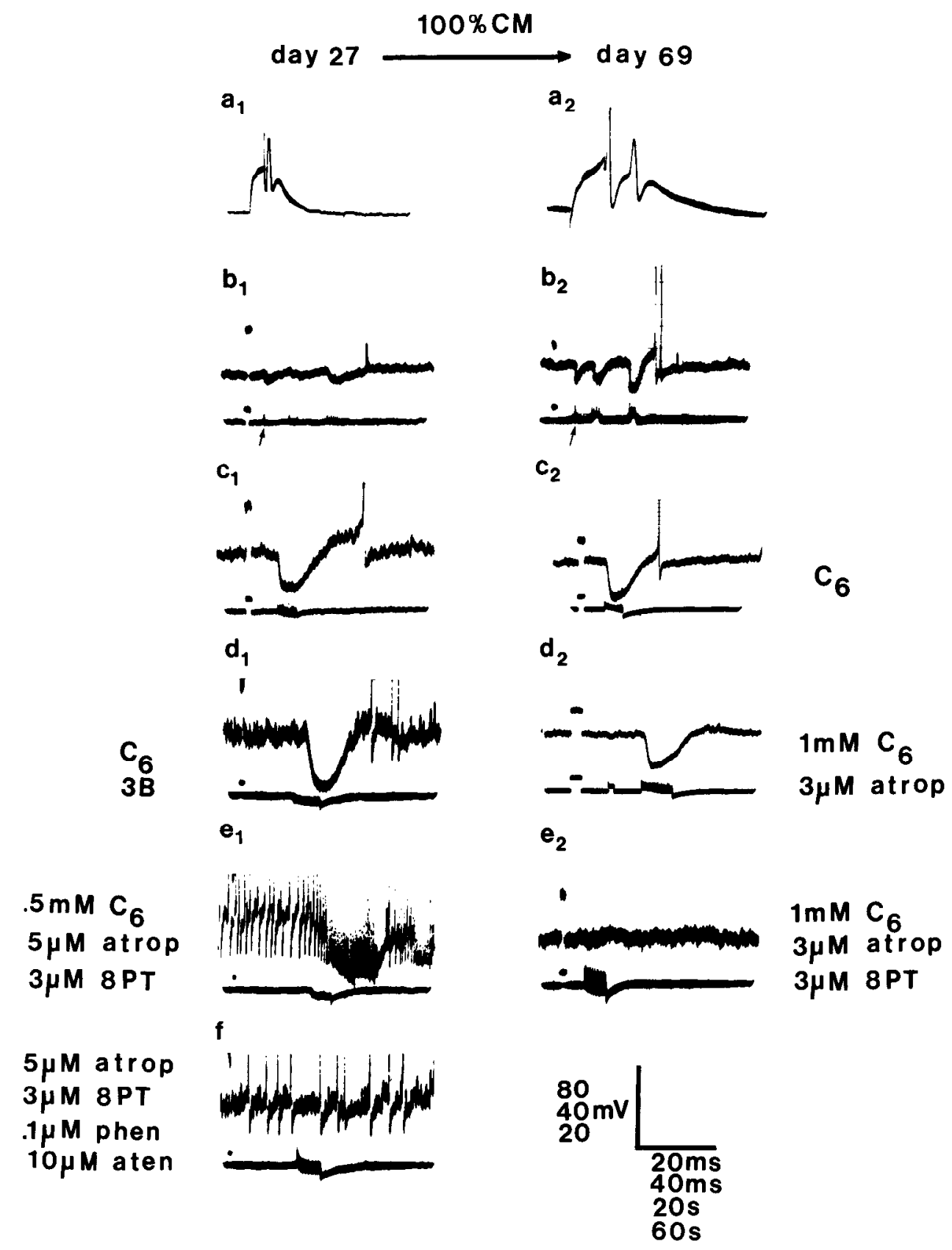

Figure 5. Serial assay of a solitary, neonate-derived neuron. The neuron was grown for $27 \mathrm{~d}$ in high-K+ medium; its status on day 27 is shown in $a_{1}-f$. Thereafter, the microculture was grown in $100 \% \mathrm{CM}$ until the second assay on day $69\left(a_{2}-e_{2}\right)$. The neuron was adrenergic/cholinergic/ purinergic on day 27. Cholinergic function is shown by the large autaptic EPSP that follows the neuronal impulse in $a_{1}$. In all other records except $a_{2}$, a brief $10 \mathrm{mV}$ calibration pulse appears near the beginning of the myocyte and neuronal traces. In $b_{1}$, a single neuronal impulse (arrow) gave rise to a hyperpolarization of the myocytes of about $1 \mathrm{mV}$, and a train of impulses at $1 \mathrm{~Hz}$ was slightly more effective. In $c_{1}$, neuronal impulses at $10 \mathrm{~Hz}$ hyperpolarized the myocytes by about $7 \mathrm{mV}$, followed by a myocyte impulse. In the presence of $5 \mu \mathrm{M}$ atropine sulfate (50x the usual blocking concentration), $0.1 \mu \mathrm{M}$ phentolamine and $10 \mu \mathrm{M}$ atenolol ("triple blocker" or $3 \mathrm{~B}$ ) plus $0.5 \mathrm{~mm}$ hexamethonium $\left(C_{6} ; d_{1}\right)$, neuronal activity at $20 \mathrm{~Hz}$ still produced a large, but more slowly rising, hyperpolarization (note that sweep speed in $c_{1}$ is $3 \times$ that in $d_{1}$ ); this disappeared in the presence of 8-phenyltheophylline $\left(8 P T ; e_{1}\right)$, indicating a purinergic origin. The larger amplitude of the hyperpolarization in $d_{1}$ is presumably due to block of adrenergic effects that partly coincide with the response in $c_{1}$. In $e_{1}, 5 \mu \mathrm{M}$ atropine and 8PT (nominally $3 \mu \mathrm{M}$ ), plus $0.5 \mathrm{~mm} \mathrm{\textrm {C } _ { 6 }}$, blocked the cholinergic and purinergic hyperpolarizations, leaving only a pronounced adrenergic excitation (high-frequency impulses that are not individually resolved but repolarized to more negative levels). This excitation was blocked $(f)$ by adding $0.1 \mu \mathrm{M}$ phentolamine and $10 \mu \mathrm{M}$ atenolol to the atropine and $8 \mathrm{PT}$; now the neuron evoked no consistent effect on the myocytes (stimulation at $20 \mathrm{~Hz}$ ). The cholinergic, purinergic, and adrenergic effects are seen in isolation in $a_{1}, d_{1}$, and $e_{1}$. When reassayed on day $69\left(a_{2}-e_{2}\right)$, the neuron was simply cholinergic/purinergic within the sensitivity of the assay. The autaptic cholinergic effect was apparently stronger $\left(a_{2} ;\right.$ a second neuronal impulse was evoked by the autaptic EPSP) than on day $27\left(a_{1}\right)$, and the effect of a single neuronal impulse on the myocytes $\left(b_{2}\right.$, arrow) was larger (note that the amplification of the myocyte trace is half that in $\left.b_{1}\right)$. In $c_{2}$, a train of neuronal impulses at $5 \mathrm{~Hz}$ (in the presence of $1 \mathrm{~mm}$ hexamethonium) produced a hyperpolarization of the myocytes more than twice as great as in $c_{1}$ (amplification of the myocyte trace half that of $c_{1}$ ) and the frequency of stimulation was half as great. A purinergic hyperpolarization, seen in isolation in $d_{2}$ (in the presence of $3 \mu \mathrm{M}$ atropine sulfate and $1 \mathrm{mM}$ hexamethonium) and about the same size as in $d_{1}$, was produced by a lower frequency of neuronal activity $\left(5 \mathrm{~Hz}\right.$ in $\left.d_{2}\right)$. When the cholinergic and purinergic effects were blocked in the presence of atropine and 8PT (plus hexamethonium), no excitatory, adrenergic effect could be detected. While the cholinergic effect was now apparently stronger (e.g., $a_{1}$ vs $a_{2}$, $b_{1}$ vs $b_{2}$ ), the adrenergic effect was gone. Vertical scale: $80 \mathrm{mV}$ for $a_{2}$ and myocyte traces $b_{2}, c_{2}, d_{2} ; 20 \mathrm{mV}$ for the myocyte traces $b_{1}-f, e_{2}$. Horizontal scale: $20 \mathrm{msec}$ for $a_{2} ; 40 \mathrm{msec}$ for $a_{1}, 20 \mathrm{sec}$ for $b_{1}, c_{1}, c_{2}, d_{2} ; 60 \mathrm{sec}$ for the remaining traces. 

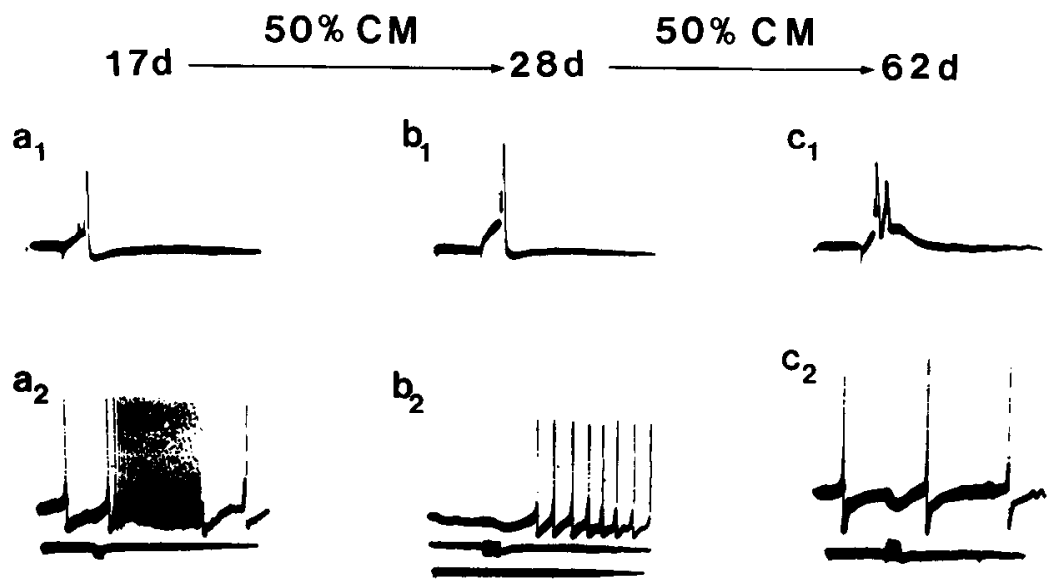

$\mathbf{b}_{3}$
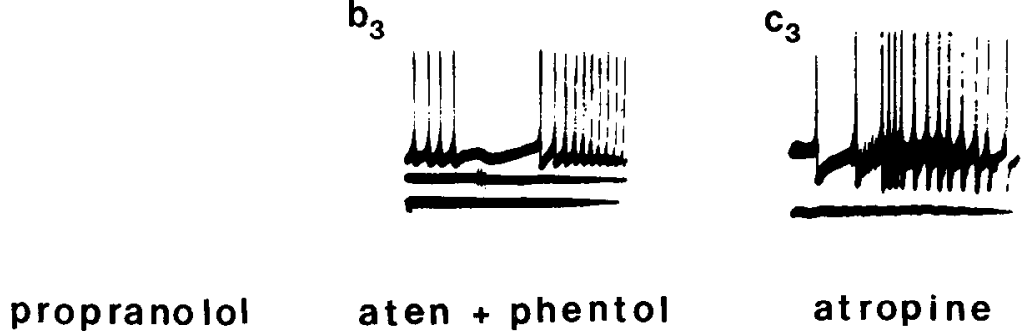

atropine

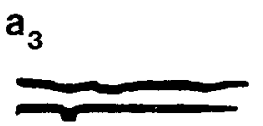

$\mathbf{b}_{\mathbf{4}}$

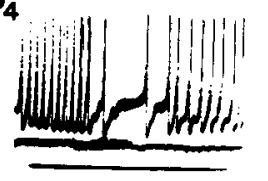

$b_{5}$

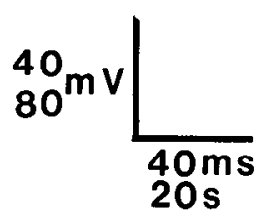

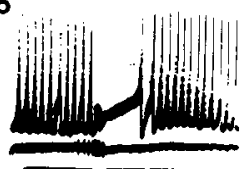

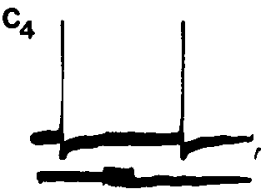

Figure 6. Serial assay of a solitary neonate-derived neuron that underwent a transition from apparently purely adrenergic status to apparently purely cholinergic status by way of dual function. The solitary neuron was grown in high-K $\mathrm{K}^{+}$medium for $17 \mathrm{~d}$. On day $17 \mathrm{it} \mathrm{had} \mathrm{no} \mathrm{autaptic} \mathrm{effect}$ $\left(a_{1}\right)$. It exerted a strongly excitatory effect on the myocytes $a_{2}$; neuronal impulses at $20 \mathrm{~Hz}$ for about 2 sec) that was blocked by $1 \mu \mathrm{M}$ propranolol $\left(a_{3}\right)$; on four trials like that of $a_{3}$ there was no stimulus-locked effect of the neuron on the unsteady myocyte membrane potential. The culture was returned to the incubator and fed $50 \% \mathrm{CM}$ to promote cholinergic function. It was reassayed on day 28 . There was no autaptic effect $\left(b_{1}\right)$. However, a train of neuronal impulses at $50 \mathrm{~Hz}$ for about $3 \mathrm{sec}$ now produced a dual effect $\left(b_{2}\right)$ that was mimicked by three brief puffs of $20 \mu \mathrm{M}$ bethanechol (a muscarinic agonist) plus $10 \mu \mathrm{M} \mathrm{NE}\left(b_{3}\right)$; the puffs are marked by the deflections of the middle trace. In the presence of $10 \mu \mathrm{M}$ atenolol and 0.1 $\mu \mathrm{M}$ phentolamine, the neuronal $\left(b_{4}\right)$ and agonist-induced $\left(b_{5}\right)$ effects were purely inhibitory (neuronal impulses at $50 \mathrm{~Hz}$ for $10 \mathrm{sec} ; 10$ puffs marked by deflections of the middle trace). After another $34 \mathrm{~d}$ in $50 \% \mathrm{CM}$, the neuron was reassayed. It now had a pronounced autaptic effect $\left(c_{1}\right)$, and the effect of a train of impulses on the myocytes $\left(c_{2} ; 20 \mathrm{~Hz}\right.$ for about $\left.2 \mathrm{sec}\right)$ was simply inhibitory. An excitatory response to puffs of $5 \mu \mathrm{M}$ NE $\left(c_{3}\right)$ demonstrated that the myocytes were still responsive to NE (puffs marked by artifactual deflection of the myocyte trace after the second impulse). As $0.2 \mu \mathrm{M}$ atropine sulfate blocked the neuronally evoked hyperpolarization without unmasking a further ncuronal effect $\left(c_{4}\right)$, it appears that the neuron was now purely cholinergic. Vertical scale: $80 \mathrm{mV}$ for $a_{1}, b_{1}, c_{1}, b_{2}, b_{3}, c_{4} ; 40 \mathrm{mV}$ for all other myocyte traces. Horizontal scale: 40 msec for $a_{1}, b_{1}, c_{1} ; 20 \mathrm{sec}$ for all other traces. Some myocyte impulses were retouched for clarity.

( $c_{1}$ vs $d_{1}$ ). A purinergic contribution to this response is seen in isolation in $d_{1}$. An adrenergic contribution is seen in isolation in $e_{1}$ in the presence of atropine and the purinergic blocker 8-PT. A combination of these blockers eliminated the response to neuronal activity $(f)$ and established this neuron as adrenergic/ cholinergic/purinergic in status on day 27 . Within the sensitivity of the re-assay on day 69 , the adrenergic effect was nil $\left(e_{2}\right)$, while the cholinergic effect was apparently stronger: The larger myocyte response to a single neuronal impulse or brief train in $b_{2}$ was apparently mainly cholinergic in origin, as a similar brief train in the presence of atropine (second deflection of neuronal trace in $d_{2}$ ) had little effect on the myocytes. The purinergic effect was apparently stronger; a hyperpolarization of the myocytes $\left(d_{2}\right)$ of about the same size as in $d_{1}$ was evoked by a lower frequency of neuronal activity. On day 69 the neuron was simply cholinergic/purinergic in status, within the sensitivity of the assay. The increased cholinergic effects at autaptic $\left(a_{2}\right)$ and cardiac junctions $\left(b_{2}\right)$ might have been caused by a variety of preor postsynaptic changes, individually or in combination, such as an increase in the number of junctions, in the intensity of cholinergic metabolism or number of vesicles in each terminal or in the concentration of postsynaptic receptors. Of particular relevance here is that the cholinergic effect increased in intensity, while the adrenergic effect disappeared.

An apparently complete transition is illustrated in Figure 6. On day 17 the neuron lacked an autaptic effect, like some other neurons grown in high- $\mathrm{K}^{+}$medium, and exerted an excitatory effect $\left(a_{2}\right)$ that was eliminated by propranolol $\left(a_{3}\right)$. On day 28 

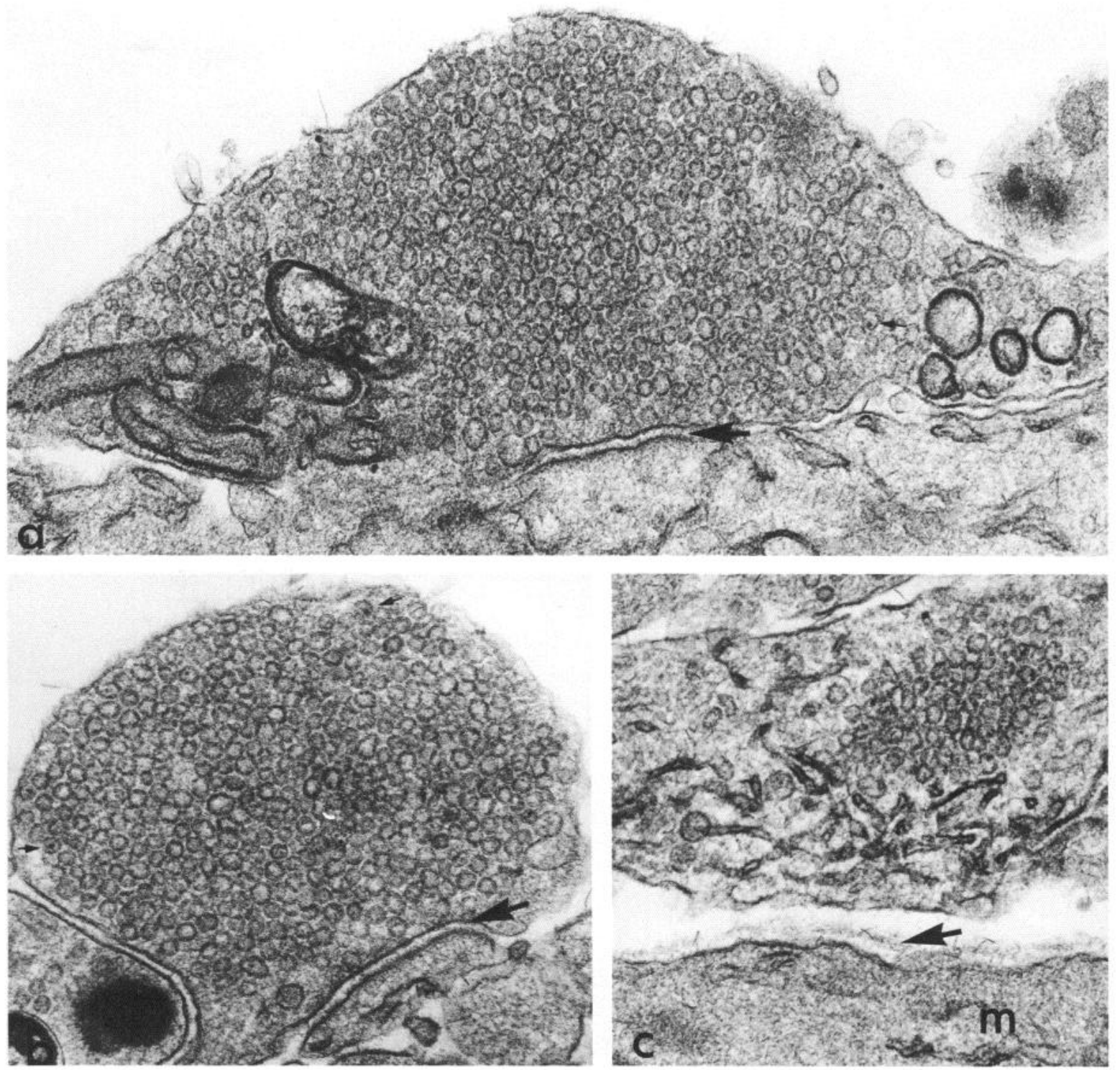

Figure 7. Terminals from the serially assayed neuron of Figure 6 . After the last assay, the neuron was incubated with $10^{-5} \mathrm{M} 5$-hydroxydopamine for 30 min. $a$ and $b$, Autapses. $c$, Varicosity on the myocytes. Most of the vesicles are clear, but a small proportion (<1\%) contains precipitate (SGV; small arrows in $a$ and $b$ ). This is consistent with the idea that although the neuron was apparently purely cholinergic in function, it had not completely lost the ability to take up and store the exogenous catecholamine. Large arrows in $a$ and $b$ indicate synaptic specializations; in $c$, the large arrow indicates the basal lamina that coats the myocyte surface. Magnification, $\times 44,000(a-c)$.

the neuron exerted a clear dual effect $\left(b_{2}\right)$ that was mimicked by puffs of a muscarinic agent, bethanechol, plus NE $\left(b_{3}\right)$. On day 62 , the neuron exhibited a strong autaptic effect $\left(c_{1}\right)$ and an apparently purely inhibitory effect on the myocytes $\left(c_{2}\right)$ that was eliminated by atropine $\left(c_{4}\right)$. The myocytes were still sensitive to puffs of NE $\left(c_{3}\right)$, so that an excitatory response to neuronal activity would have been expected (as in $a_{2}$ and $b_{2}$ ) if the neuron still secreted a significant amount of NE. The change in status of the neuron is best seen by comparing $a_{2}$ with $c_{2}$.

After the physiological assay, this neuron was incubated for $30 \mathrm{~min}$ in medium that contained $10 \mu \mathrm{M}$ 5-hydroxydopamine, an analog of NE known to be taken up and stored in SGV by adrenergic neurons (Tranzer et al., 1969); the intent was to see whether a neuron that was apparently purely cholinergic in function detectably retained this property of adrenergic neurons. Figure 7 shows the synaptic fine structure of this neuron. A small proportion of the vesicles ( $<1 \%$ on average) in autapses or junctions with myocytes contained granular precipitate (small arrows in $a$ and $b$ ). Note the size of the terminals and the dense packing of the vesicles, in comparison with the terminals of younger neurons in Figure 3.

Although the total number of neonate-derived neurons that showed a change was small (14), the serial assays provided direct evidence for the adrenergic-to-cholinergic transition. While the serial assay method can only set an upper limit on the transition time in a single neuron (it is plausible that the actual changes occurred in intervals shorter than the intervals between assays), the experiments of Figure 4 are all consistent with rather slow transitions. In the case of the five neurons of Figure $4 c$, the total transition times would obviously have been longer than the 4$15 \mathrm{~d}$ taken to go from $\mathrm{A} / \mathrm{C}$ to $\mathrm{A} / \mathrm{C}^{+}$; similar considerations apply to the neurons of Figure 4, $a$ and $b$, that failed to complete the full transition in 4-31 d. Landis and Keefe (1983) reported a transition time of roughly 2 weeks in the developing sympathetic innervation of sweat glands in vivo.

The apparent heterogeneity in the rate of the transitions is of interest. The neurons of Figure $4 a$ did not change status detectably over intervals of 4-14 d; a detectable change occurred 

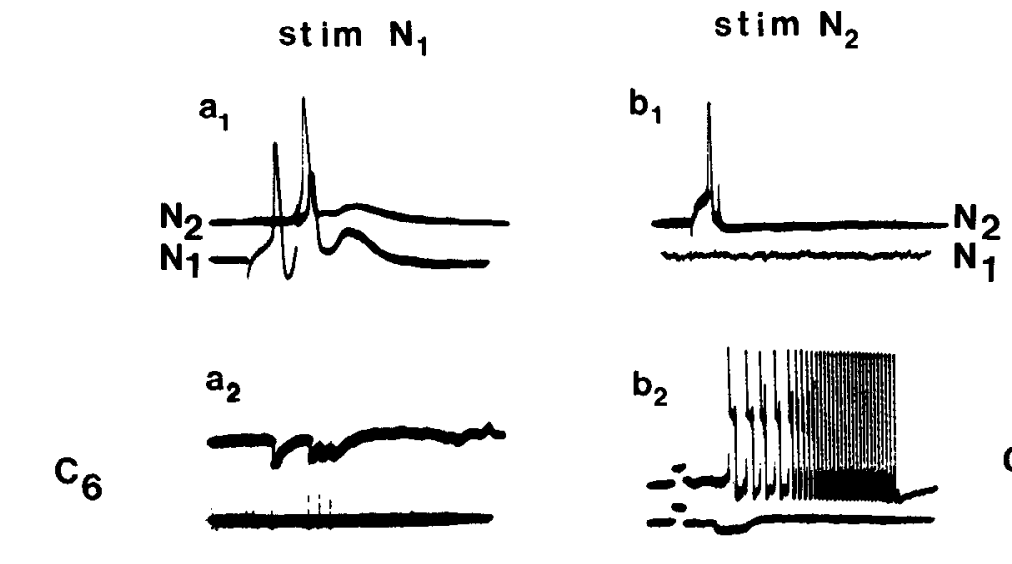

\section{$\mathrm{C}_{6}$}

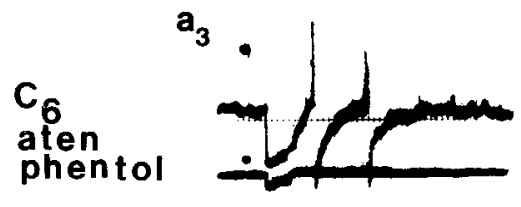

$b_{3}$

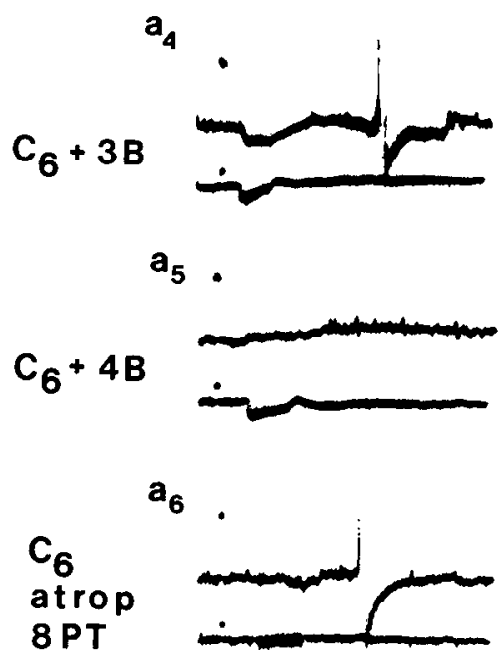

$\mathbf{C}_{6}$ aten phentol

Figure 8. Two neonate-derived neurons that had different transmitter status after $80 \mathrm{~d}$ in the same microculture. Neuron $\mathrm{N}_{1}$ was examined on days 76 and 80; no change in status was observed. The records in $a_{1}$ and $a_{2}$ were taken on day 76 , the other records on day 80 . Status of $N_{1}, \mathrm{~A}$ directly evoked impulse in $N_{1}\left(a_{1}\right.$, lower trace) produced synaptically evoked impulses followed by EPSPs in both neurons; this shows that $N_{1}$ was at least cholinergic. In the presence of $1 \mathrm{~mm}$ hexamethonium $\left(C_{6} ; a_{2}\right)$ added to block interaction between $\mathrm{N}_{1}$ and $\mathrm{N}_{2}$, single impulses in $\mathrm{N}_{1}$ (deflections of lower trace) produced hyperpolarizations of the myocytes (upper trace) followed by a small depolarization. In $a_{3}$, the perfusion fluid contained $1 \mathrm{mM} \mathrm{C}, 30 \mu \mathrm{M}$ atenolol, and $0.1 \mu \mathrm{M}$ phentolamine; the first deflections of the upper and lower traces, as in all the other records except $b_{1}$, are brief calibration steps of $10 \mathrm{mV}$. A train of neuronal impulses $(20 \mathrm{~Hz}$ for about $12 \mathrm{sec}$ ) evoked a hyperpolarization followed by two myocyte impulses. In $a_{4}, 10 \mu \mathrm{m}$ atropine sulfate added to the other blockers $\left(C_{6}+3 B\right)$, sharply diminished the hyperpolarization, showing that most of it was due to secretion of ACh by $\mathrm{N}_{1}$. In $a_{3}$, the remaining atropine-resistant hyperpolarization was blocked by addition of 8-phenyltheophylline ( $8 P T$, nominally $3 \mu \mathrm{M} ; C_{6}+4 B$ ) and was therefore a purinergic effect of $\mathrm{N}_{1}$, as described in Furshpan et al. (1986b). The remaining depolarization is the NAE effect described in Matsumoto et al. (in press); on 1 trial out of 5, a myocyte impulse was evoked (not shown). When the two adrenergic blockers were removed $\left(a_{6}\right.$; presence of $\mathrm{C}_{6}$, atropine, and $\left.8 \mathrm{PT}\right)$, the same neuronal activity ( $20 \mathrm{~Hz}$ for $24 \mathrm{sec}$ ) evoked myocyte impulses on four of five trials, consistent with a very weak adrenergic effect. Thus, $\mathrm{N}_{1}$ was cholinergic/purinergic/NAE and possibly adrenergic. Status of $N_{2}$, An impulse in $\mathrm{N}_{2}$ $\left(b_{1}\right.$, upper trace) had no autaptic component and no effect on $\mathrm{N}_{1}$ (lower trace). In $b_{2}$, a train of impulses in $\mathrm{N}_{2}$ ( $20 \mathrm{~Hz}$ for 5 sec) evoked a train of myocyte impulses. The concentration of $C_{6}$ was $1 \mathrm{mM}$. When $30 \mu \mathrm{M}$ atenolol and $0.1 \mu \mathrm{M}$ phentolamine were added $\left(b_{3}\right)$, the excitatory effect was blocked (stimulation at $20 \mathrm{~Hz}$ for $9 \mathrm{sec}$ ), and no consistent neuronal effect was unmasked. Thus, $\mathrm{N}_{2}$ was apparently purely adrenergic. Vertical scale: $20 \mathrm{mV}$ for upper traces in $a_{3}-a_{6}, b_{3} ; 40 \mathrm{mV}$ for upper trace in $a_{2}$, lower trace in $b_{1}, 80 \mathrm{mV}$ in all other traces. Horizontal scale: $20 \mathrm{msec}$ for $a_{1} ; 40 \mathrm{msec}$ for $b_{1} ; 20 \mathrm{sec}$ for $a_{2}, b_{2} ; 60 \mathrm{sec}$ for other records.

in six other neurons (not fed CM) over intervals of 6-15 d. Further evidence for heterogeneity in transition rates is given in the next section.

It is noteworthy that all of the neurons of Figure 4 expressed cholinergic function by the final assay, if they had not done so earlier, except for the third neuron of Figure $4 a$. Although the null state of this neuron may well have been pathological, it is included in Figure 4 to acknowledge several other possibilities: that some neurons secrete transmitters having little electrical effect on the myocytes or the neurons themselves (e.g., a pep- 
tidc); that some neurons undergo the transition by way of a null state; or that some neurons are unable to accept cardiac myocytes as target cells. It is also noteworthy that none of the neurons in dual status reverted to adrenergic status during the serial assays, i.e., the transition was unidirectional. Finally, the nine neurons of Figure $4, b, d$, and $e$, either gained a new transmitter function or lost an old one, within the sensitivity of the assays (this point is considered further in the Discussion).

Does addition of CM to the medium hasten the transition or simply initiate a transition whose time course is otherwise controlled? Landis (1980) has provided strong evidence that in mass cultures the fine structure of terminals changes more quickly in the presence of a high concentration of CM than in less "cholinergic" culture conditions. It is of interest that the shortest interval in which there was a detectable change $(4 \mathrm{~d}$, second neuron of Fig. $4 c$ ) occurred in the presence of $50 \% \mathrm{CM}$ and that four of the five serially assayed neurons that achieved apparently purely cholinergic status did so in the presence of 50 or $100 \%$ CM (Fig. 4, $d$ and $e$ ).

\section{Further evidence for heterogeneity in the timing of the adrenergic-to-cholinergic transition: neonate-derived neurons assayed only once}

One possible cause of the apparent heterogeneity of the serially assayed neurons is differences between the microcultures in the number or character of the cocultured cardiac cells (see Materials and Methods in Furshpan et al., 1986a); heterogeneity after the first assay might also have been caused by different degrees of damage produced by the prolonged impalements during the first assays. These possible sources of heterogeneity were not present when two or more neurons grown in the same microculture were assayed only once. In multiple-neuron microcultures, the neurons were sometimes conspicuously different in status. An example is shown in Figure 8.

The microculture of Figure 8 contained two neurons, $N_{1}$ and $N_{2} . N_{1}$ evoked suprathreshold EPSPs in $N_{2}$ and in itself $\left(a_{1}\right)$, and was therefore at least cholinergic; $\mathrm{N}_{2}$ had no observable synaptic effect on either $N_{1}$ or itself $\left(b_{1}\right)$. To permit the effects of each neuron on the myocytes to be independently observed, all other records were made in the presence of a high concentration of hexamethonium that completely suppressed the cholinergic EPSPs (not shown). Within the sensitivity of the assay, $\mathrm{N}_{1}$ was relatively strongly cholinergic/moderately purinergic/ NAE and perhaps very weakly adrenergic; in four of five trials, like that of $a_{6}$, a myocyte impulse was evoked in the absence of adrenergic blockers, but in only one of five trials, like that of $a_{5}$, was a myocyte impulse evoked when the adrenergic blockers were present. This possibly adrenergic effect was clearly at the limit of sensitivity of the assay. In contrast, $\mathrm{N}_{2}$ exerted a substantial excitatory effect in the myocytes $\left(b_{2}\right)$ that was completely blocked by adrenergic blockers $\left(b_{3}\right)$ without unmasking any other neuronal effect. Thus, on day 80 , while $N_{1}$ had apparently almost completed the adrenergic-to-cholinergic transition, $\mathrm{N}_{2}$ had presumably not begun it. As noted earlier, in this microculture the effect(s) evoked by each neuron established that the (coupled) myocytes were sensitive to an effect evoked weakly or not at all by the other neuron. The heterogeneity of adrenergic/cholinergic status in such pairs of microcultured neurons was present at first assay and therefore cannot have been an artifact induced by an earlier impalement. Several possible causes of this heterogeneity are considered in the Discussion.

Further evidence for heterogeneity in the timing of the transition came from single assays on solitary neurons. For example, adrenergic/cholinergic dual status was observed as early as $9 \mathrm{~d}$ (first two neurons of Fig. $4 a$ ) and in some cases was already well advanced in the second week; the oldest neonate-derived neuron that displayed adrenergic/cholinergic dual status was 15 weeks in microculture (not illustrated). The youngest neuron that ex- hibited apparently purely cholinergic function was $12 \mathrm{~d}$ old, but this assay was not particularly stringent. A number of neurons failed to exhibit cholinergic function after more than 6 weeks in culture (see below and Fig. 9a); two of these (one is neuron $\mathrm{N}_{2}$ of Fig. 8) appeared to be purely adrenergic during the 12 th week. In the case of neuron $\mathrm{N}_{2}$ in Figure 8 , it is clear from the status of $\mathrm{N}_{1}$ that the microculture environment was capable of inducing the adrenergic-to-cholinergic transition and that the myocytes were sensitive to ACh. This raises the possibility that some neurons of the neonatal superior cervical ganglion are not plastic in the culture conditions employed or that some revert to apparently purely adrenergic status after entering the transition. However, most neonate-derived neurons that were assayed only once exhibited statuses that were intermediate between these extremes, as described in the next section.

\section{Incidence of cholinergic and adrenergic functions in singly assayed neonate-derived neurons}

A week-by-week census of 372 neonate-derived neurons is shown in Figure $9 a$. No assays were made during the first week in culture. Figure $9 a$ includes all assays in which a judgment about the presence or absence of cholinergic function could be made. Four considerations affect the interpretation of this data. First, 95 of the neurons were characterized as at-least-cholinergic solely on the basis that the neuron formed cholinergic synapses on itself or a neighboring neuron in the same microculture, but 26 other cases in which the only information was a lack of such synaptic effects were excluded, because no definite conclusion could be reached with respect to cholinergic function. This exclusion tends to overestimate the proportion of neurons that displayed cholinergic function; some of these neurons probably lacked cholinergic function. Second, we excluded another 21 neurons that also lacked an autaptic effect but produced a myocyte hyperpolarization whose sensitivity to atropine or 8-PT was not checked. This exclusion tends to underestimate the proportion of at-least-cholinergic neurons; many of these cases were probably cholinergic. Third, neurons were classified as notdetectably-cholinergic if the assays on the myocytes were reasonably stringent, but in some cases a weak cholinergic effect may have been overlooked; again, this probably underestimates the proportion of at-least-cholinergic neurons. Fourth, 18 of the neurons were from a plating described in Furshpan et al. (1986b) in which purinergic function was unusually marked and cholincrgic function unusually rare, for unknown reasons. We tentatively conclude that Figure $9 a$ represents reasonably well the incidence of cholinergic function in the microculture conditions used, at least during the first 7 weeks when substantial numbers of cultures were studied.

Of the neurons summarized in Figure $9 a$, about $78 \%(292)$ displayed at-least-cholinergic function. Only $8 \%$ (23) were apparently purely cholinergic (hatched bars). Among the at-leastcholinergic cases were neurons that also displayed adrenergic, purinergic, or NAE functions. About $45 \%$ (131) of the at-leastcholinergic neurons also exhibited adrenergic function; this is a lower limit, because for 95 of the at-least-cholinergic group, the only test of function was the presence of neuron-neuron synapses; probably most of the 95 neurons were also adrenergic/ cholinergic in status. If these 95 neurons are excluded, $66 \%$ of the remaining 197 neurons were (at least) adrenergic/cholinergic in status.

The percentage of neurons that displayed at-least-cholinergic function in each of the second to seventh weeks is shown in Figure $9 \mathrm{c}$. The high incidence of this status, rising to $98 \%$ of assays in the sixth week, in neurons grown in the normal medium (the curve marked $C$ ) is consistent with other evidence from mass cultures and microcultures (discussed above) for a transition toward cholinergic status and with the idea that most of the microcultured neurons are capable of assuming cholin- 

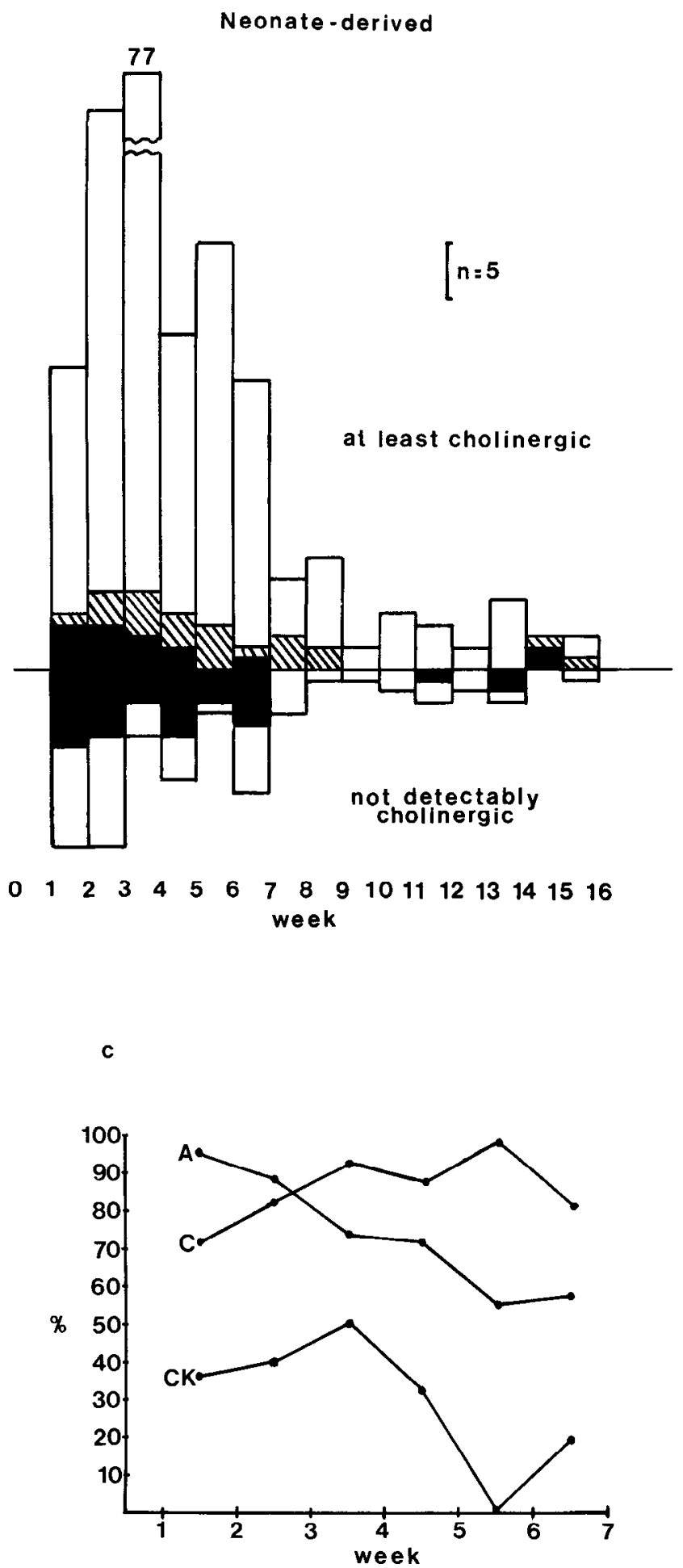

ergic function. However, the microculture conditions used did not induce all neurons to become cholinergic or more than a minority to become apparently purely cholinergic. While the number of assayed neurons fed high- $\mathrm{K}^{+}$medium was relatively small (solid bars in Fig. 9a), a comparison of curves C and CK in Figure $9 c$ suggests that this medium deterred expression of cholinergic function, as in mass cultures (Walicke et al., 1977).

Figure $9 b$ shows a similar week-by-week census of 214 neurons whose assays were a reasonably sensitive assessment of adrenergic function; however, some neurons categorized as notdetectably-adrenergic may actually have expressed adrenergic b

Neonate-derived

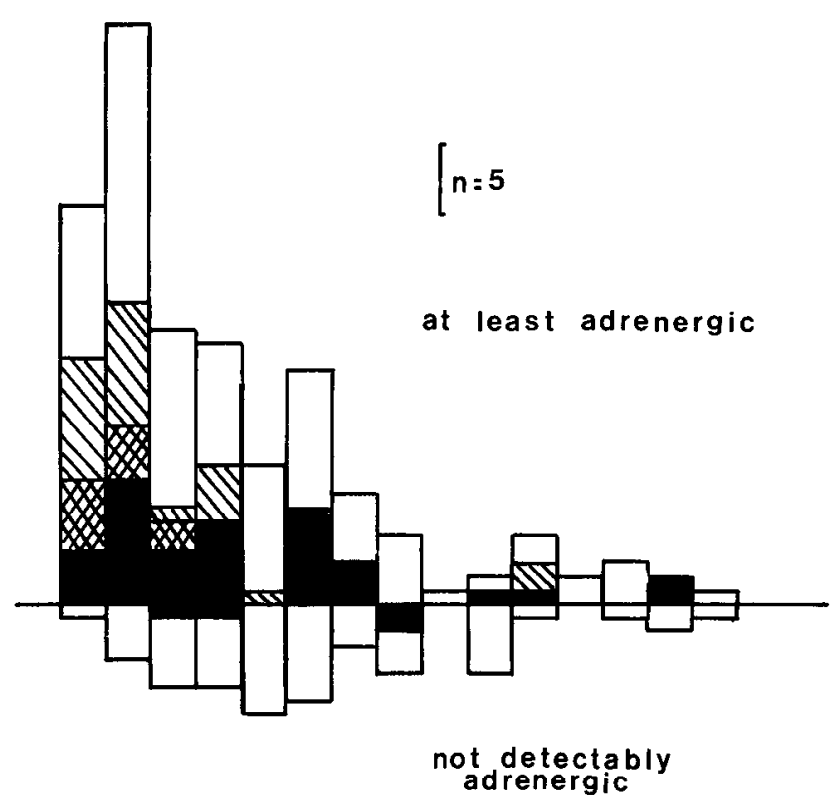

$\begin{array}{llllllllllllllll}0 & 1 & 2 & 3 & 4 & 5 & 6 & 7 \underset{\text { week }}{8} & 9 & 10 & 11 & 12 & 13 & 14 & 15 & 16\end{array}$

Figure 9 . Incidence of cholinergic and adrenergic functions in neonatederived neurons, assayed once at various culture ages. In $a-c$, assays during a particular week indicated on the horizontal axis wcrc pooled (e.g., assays done on days 8-14 are plotted between week 1 and week 2 ); in $a$ and $b$, the scale represents 5 assays. The day of entry into culture is denoted as week 0 . No assays were done during the first week in culture. In $a$, a total of 372 assays is summarized; the criteria for including these assays are described in the text. Apparently-purely cholinergic neurons are represented by hatched bars, and neurons grown in high-K $\mathrm{K}^{+}$medium are represented by solid bars. The percentage of atleast-cholinergic neurons during each of the second through seventh weeks is shown in $c$; neurons grown in normal medium are represented by curve $C$, and neurons grown in high- $\mathrm{K}^{+}$medium are represented by curve $C K$. Apparently, high- $\mathrm{K}^{+}$medium deterred expression of cholinergic function, as it deters expression of synthesis and storage of ACh in mass cultures (Walicke et al., 1977). In $b, 216$ assays of adrenergic function are summarized; this includes many of the neurons summarized in $a$ but excludes cases in which neuron-neuron interaction was the only assay and adds additional cases where adrenergic function, but not cholinergic function, was assessed. Solid and cross-hatched bars; Neurons grown in high- $\mathrm{K}^{+}$medium. Cross-hatched and hatched bars; Neurons that were apparently purely adrenergic. The percentage of atleast-adrenergic neurons, grown in normal medium and assayed in the second through seventh weeks, is shown as curve $A$ in $c$.

function very weakly, and the data probably underestimate the incidence of this status. The difficulty in interpreting borderline cases is illustrated by neuron $N_{1}$ of Figure 8 ; this cell was categorized as at-least-adrenergic in constructing Figure $9 b$. About $77 \%$ of the neurons of Figure $9 b$ were at-least-adrenergic. Apparently purely adrenergic neurons are represented by hatched bars in $b$ (cross-hatched if the neuron was grown in high- $\mathrm{K}^{+}$ medium); although the total number of such neurons was not large, their incidence appears to fall with increased age in microculture. Moreover, subject to the uncertainty mentioned above about neurons that apparently lacked adrenergic function, the 
Figure 10. Adrenergic/cholinergic dual function in an adult-derived solitary neuron. The neuron was isolated from an 8-week-old rat and grown in microculture for $19 \mathrm{~d}$. Like most other adult-derived neurons, this neuron lacked an autaptic effect $(a)$. A train of neuronal impulses $(20 \mathrm{~Hz}$, as in all other records) evoked a dual effect in the myocytes $(b)$. In the presence of $0.2 \mu \mathrm{M}$ atropine sulfate, the neuronally evoked hyperpolarization was blocked $(c)$; the remaining excitatory effect on the myocytes was partially blocked by adding $1 \mu \mathrm{M}$ propranolol to the atropine solution (d). Thus, the excitatory effect was at least partially adrenergic in origin. Removal of the two blocking agents restored the original dual effect of the neuron (e). Vertical scale: $40 \mathrm{mV}$ for $b$ $e ; 80 \mathrm{mV}$ for $a$. Horizontal scale: 40 msec for $a ; 4 \mathrm{sec}$ for the other records. a

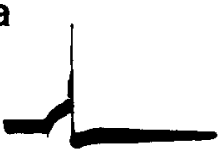

C

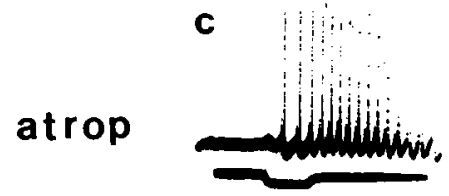

e

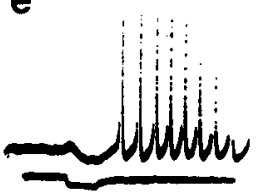

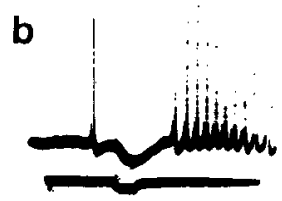

d

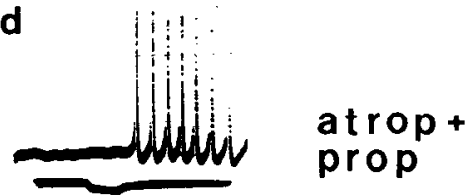

proportion of neurons that were detectably at-least-adrenergic also fell during the second through the seventh weeks; this is plotted as curve A in Figure $9 c$, for neurons grown in normal medium. This is consistent with other evidence from mass cultures and microcultures grown in strongly "cholinergic" conditions that adrenergic expression generally declines as cholinergic expression is enhanced (see above, and Landis, 1980; Patterson and Chun, 1977b; Wolinsky and Patterson, 1983; compare Johnson et al., 1980). However, it is important to note that a decline in adrenergic expression was not universal; among the at-least-adrenergic singly assayed neurons at culture ages older than 7 weeks were some that expressed relatively strong adrenergic function in company with one or more of the other transmitters, in addition to the two apparently purely adrenergic neurons of the 12th week (see Furshpan et al., 1986b; Matsumoto et al., in press).

The number of neonate-derived neurons assayed at culture ages greater than 7 weeks was too small to permit reliable conclusions about the incidence of adrenergic and cholinergic functions, beyond the obvious facts that not all neurons acquired cholinergic function or lost adrenergic function after several months in culture.

\section{Adrenergic/cholinergic dual function in adult-derived neurons}

The fact that some neonate-derived neurons expressed adrenergic/cholinergic dual function at culture ages greater than 6 weeks (a postnatal age at which rats become sexually mature) raised the question whether neurons derived from ganglia of intact rats of comparable age may also display dual status. Cholinergic states had been reported in cultured adult neurons by Wakshull et al. (1979a, b). With a method similar to that devised by Johnson (1978; see Furshpan et al. 1986a), principal neurons were obained from rats 8 weeks to about 1 year old. Of the 81 such neurons assayed in microcultures, all but 5 expressed atleast-adrenergic function ( 1 of the 5 was the apparently purely cholinergic neuron illustrated in Fig. 7 of Furshpan et al., 1986a). Twenty-two of the 81 neurons expressed both adrenergic and cholinergic functions; of these, 13 were simply adrenergic/cholinergic and the others had more complex statuses described in Matsumoto et al. (in press). An example of combined adrenergic and cholinergic function is shown in Figure 10. The neuron exerted a dual effect $(b)$, sensitive to atropine and propranolol, although the excitatory effect was not completely blocked by the concentration of propranolol employed; as the residual excitatory effect was not examined further, it is not known whether this neuron secreted a third transmitter. Thus, this neuron displayed (at least) adrenergic/cholinergic dual status. As in the case of the neonate-derived neurons, the relative strengths of the adrenergic and cholinergic effects varied from one adultderived neuron to another.

A week-by-week census of expression of cholinergic function in 81 adult-derived neurons assayed in microcultures is shown in Figure 11. A comparison of Figure 11 with Figure $9 a$ makes it clear that the incidence of cholinergic function was considerably lower during the weeks $2-7$ in culture for adult-derived than for neonate-derived neurons.

Did adult-derived neurons of dual status acquire cholinergic function in culture (plasticity like that of neonate-derived neurons), or were they dual in status before being placed in culture? In 10 serial assays of adult-derived neurons, cholinergic function was absent at first assay; it was detected at a later assay in two cases. One of these cases, a neuron from a 41/2-to-5-month-old rat, was illustrated by Potter et al. ( $1981 \mathrm{~b}$; in that paper, the assay days were incorrectly given as 3,12 , and 32 , instead of the correct 13,22, and 32). The other case is illustrated in Figure 12 . On day 28 , two detectable effects of trains of neuronal impulses were a pronounced adrenergic excitation of the myocytes $\left(b_{1}\right)$ and a purinergic hyperpolarization $\left(c_{1}\right)$. In $d_{1}$, no atropine was present, yet the train of neuronal impulses did not evoke a cholinergic hyperpolarization. The sensitivity of the myocytes to ACh was checked with puffs of ACh $\left(e_{1}\right)$. On day 28 , the neuron was adrenergic/purinergic in status but not detectably cholinergic.

When the neuron was reassayed on day 44 , it was conspicuously cholinergic. There was an autaptic EPSP $\left(a_{2}\right.$; compare with $\left.a_{1}\right)$, and an initial hyperpolarization of the myocytes $\left(b_{2}\right.$; compare with $b_{1}$ ) blocked by atropine $\left(c_{2}\right)$. The residual excitatory effect was blocked by adrenergic antagonists $\left(d_{2}\right)$. A residual atropine-resistant hyperpolarization was still present in $d_{2}$; this was presumably purinergic in origin (compare with $c_{1}$ ), but before 8-PT could be added to the perfusion fluid the neuron was accidently destroyed, and no further information about its status was obtained. Thus, on day 44 this neuron was (at least) adrenergic/cholinergic/probably purinergic in function.

The absence of detectable cholinergic function on day 28 , 
at least cholinergic

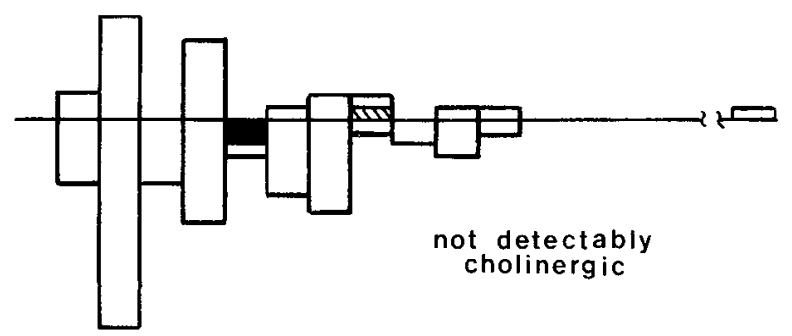

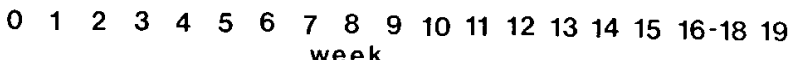

Figure 11. Incidence of cholinergic function in 81 adult-derived neurons. The scale is the same as in Figure 9a; the solid bar represents two neurons grown in high- $\mathrm{K}^{+}$medium, and the hatched bar a neuron that was apparently purely cholinergic. Comparison with Figure $9 a$ shows that the incidence of cholinergic function was lower than in neonatederived neurons.

when the neuron had formed a sufficient number of varicosities on the myocytes to exert a pronounced adrenergic effect (and a relatively weak purinergic effect), suggests that the neuron did not enter culture with appreciable cholinergic function. The subsequent appearance of conspicuous cholinergic function, in the presence of $100 \% \mathrm{CM}$, is consistent with the idea that this adultderived neuron was plastic with respect to transmitter functions. Whether the plasticity was retained from the period of perinatal development or reacquired as a result of dissociation from the ganglion and placement in culture, it was not detectable after growth in normal medium for $28 \mathrm{~d}$.

Although the number of neurons fed $\mathrm{CM}$ was small, the result was consistent with the idea that adult-derived neurons are less responsive to $\mathrm{CM}$ than neonate-derived neurons. For example, seven of the serially assayed adult-derived neurons that showed no change in status received CM for periods of $16,18,20,27$, 32,35 , and $63 \mathrm{~d}$ before the second assay; in contrast, all of the serially assayed neonate-derived neurons of Figure 4 that were fed CM for comparable periods underwent a change in status. However, at least some neurons from rats as old as 1 year may retain responsiveness to $\mathrm{CM}$; among 10 such neurons, singly assayed, 5 that received no CM displayed no detectable cholinergic function $(23,27,44,51$, and $72 \mathrm{~d}$ in cullure), while 3 of the remaining 5 that received $\mathrm{CM}$ (for 20, 20, and $72 \mathrm{~d}$ ) displayed cholinergic function (the unresponsive neurons received $\mathrm{CM}$ for 20 and $72 \mathrm{~d}$ ). Adler and Black (1984) have recently reported a decline in plasticity with respect to expression of substance $\mathrm{P}$ among neurons in explants of the superior cervical ganglion of adult rats.

\section{Synaptic interactions developed by neonate-derived neurons in the presence of tetrodotoxin and hexamethonium}

It seemed of interest to investigate whether the formation of effective junctions or the ability to display adrenergic-to-cholinergic plasticity were affected if neurons were grown chronically, from dissociation to assay, in blocking concentrations of TTX and hexamethonium. Seven neonate-derived neurons grown in medium that containcd $3.1 \mu \mathrm{M}$ TTX and $1 \mathrm{~mm}$ hexamethonium were examined; these concentrations completely block action potentials and neuron-neuron synapses ( $O$ 'Lague et al., 1978a, c). The microcultures were kindly provided by Dr. Steven Hauger. Figure 13 shows records from one of these cases.

Figure 12. Serial assays of a solitary adult-derived neuron that developed adrenergic/cholinergic function in microculture. The neuron, derived from a $350 \mathrm{gm}$ rat, was grown for $28 \mathrm{~d}$ in normal medium. At
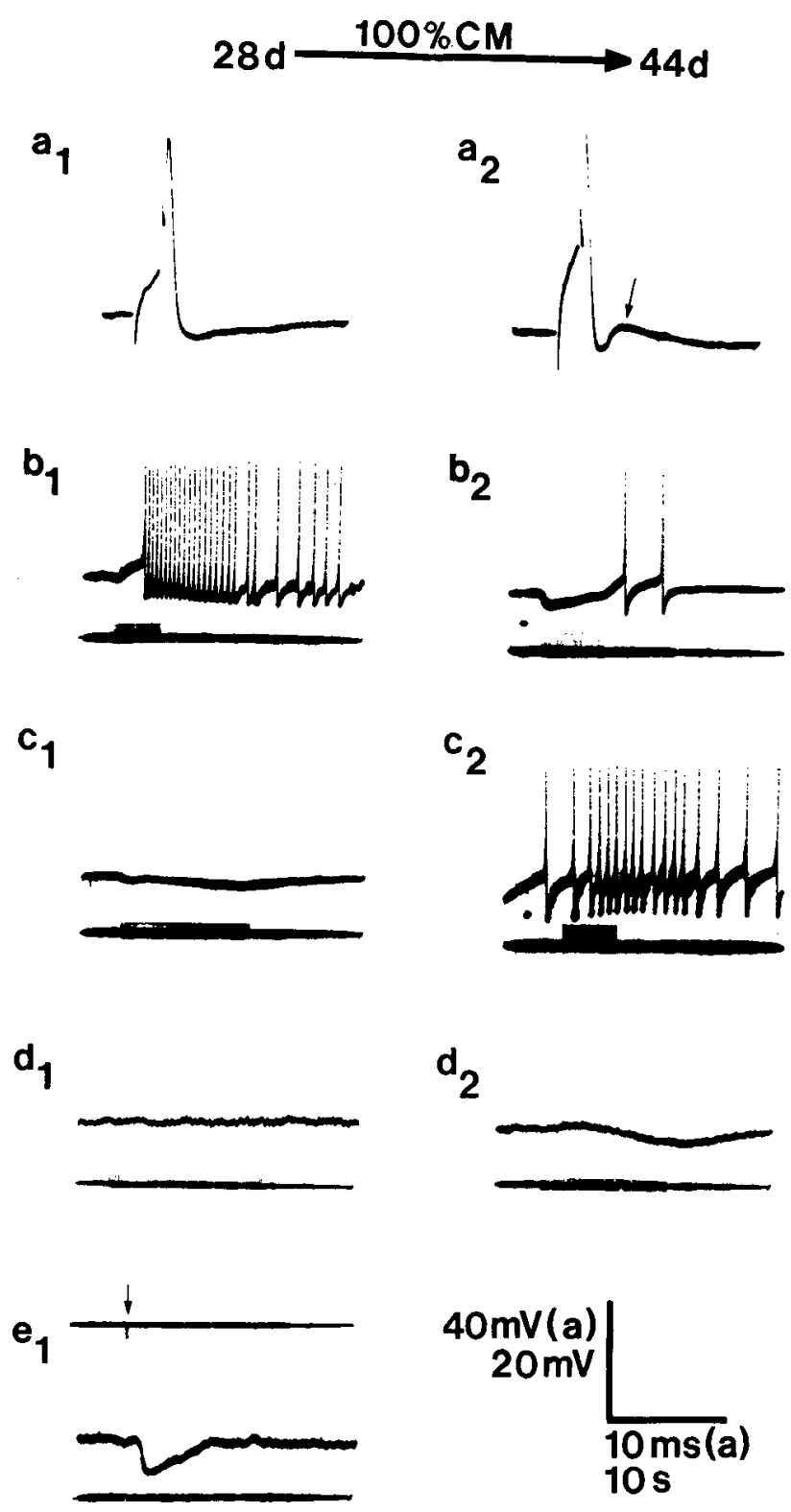

that date, it did not have a detectable autaptic effect $\left(a_{1}\right)$; its effect on the myocytes (stimulation at $20 \mathrm{~Hz}$, as in all the other records) was rather strongly excitatory $\left(b_{1}\right)$. In the presence of $0.5 \mu \mathrm{M}$ atropine, 0.1 $\mu \mathrm{M}$ phentolaminc, and $10 \mu \mathrm{M}$ atenolol, the excitatory effect was eliminated $\left(c_{1}\right)$, and a small atropine-resistant hyperpolarization was unmasked. In the absence of atropine, but with the adrenergic and purinergic effects blocked by atenolol, phentolamine, and 8PT (nominally 10 $\mu \mathrm{M})$, no cholinergic hyperpolarization was revealed in response to neuronal activity (duration about $28 \mathrm{sec} ; d_{1}$ ). That the myocytes were responsive to a moderate concentration of $A C h$ was shown with a puff of $10 \mu \mathrm{M} \mathrm{ACh}$, marked by the arrow in $e_{1}$; the myocytes were hyperpolarized by about $10 \mathrm{mV}$ (middle trace in $e_{1}$ ). Thus, on day 28 , the neuron was adrenergic/purinergic in status. The dish was returned to the incubator, fed $100 \% \mathrm{CM}$ for $16 \mathrm{~d}$, and reassayed on day 44 . There was clear evidence of cholinergic function: A substantial autaptic EPSP (arrow in $a_{2}$ ) and a dual effect on the myocytes $\left(b_{2}\right)$ in response to a train of neuronal impulses; the initial hyperpolarization of the myocytes was blocked by $0.5 \mu \mathrm{M}$ atropinc $\left(c_{2}\right)$. The remaining excitation was blocked by adding $10 \mu \mathrm{M}$ atenolol and $0.1 \mu \mathrm{M}$ phentolamine to the atropine $\left(d_{2}\right)$. An atropine-resistant hyperpolarization was unmasked $\left(d_{2}\right)$ that was probably purinergic in origin, but the neuron was accidently destroyed by the recording microelectrode before 8-PT could be added. Thus, on day 44 , the neuron was adrenergic/cholinergic/probably purinergic. Vertical scale: $40 \mathrm{mV}$ for $a_{1}, a_{2}$ and $20 \mathrm{mV}$ for all myocyte traces. Horizontal scale: $20 \mathrm{msec}$ for $a_{1}$ and $a_{2} ; 10 \mathrm{sec}$ for all other traces. 
a

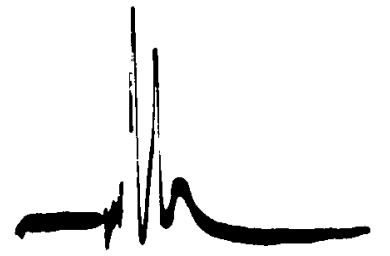

C
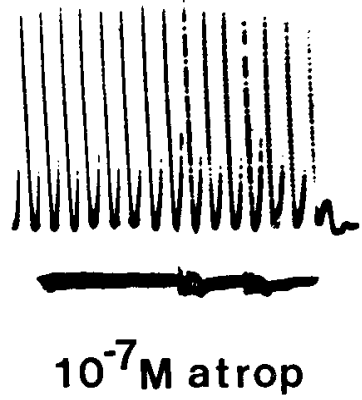

b

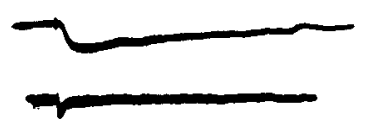

d

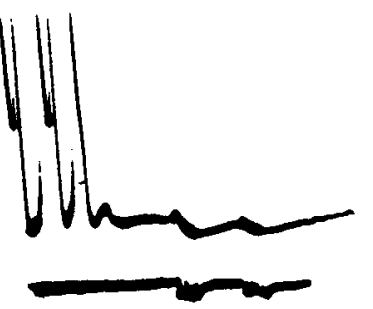

Figure 13. Synaptic function in a neuron grown chronically, before assay, in blocking concentrations of TTX and hexamethonium $\left(\mathrm{C}_{6}\right)$. After dissociation from a neonatal ganglion, the neuron was fed medium that contained $3.1 \mu \mathrm{M}$ TTX and $1 \mathrm{mM} \mathrm{C}_{b}$ for $42 \mathrm{~d}$. No action potentials or spontaneous EPSPs were present before washout of the blockers. The first suprathreshold stimulus after removal of TTX and $C_{6}$ produced the result shown in $a$; a substantial cholinergic autaptic effect was present. A single neuronal impulse produced a substantial hyperpolarization of the myocytes $(b)$. In the presence of $0.1 \mu \mathrm{M}$ atropine sulfate $(c)$, two trains of neuronal impulses ( $20 \mathrm{~Hz}$ for $0.6 \mathrm{sec}$ each) had no observable effect on the myocytes, indicating that the neuron was simply cholinergic. The inhibitory effect of neuronal activity was restored by washing out the atropine (d). Horizontal scale: $20 \mathrm{msec}$ for $a, 1 \mathrm{sec}$ for $b ; 2 \mathrm{sec}$ for $c$ and $d$.

In the presence of the two agents, no action potentials could be evoked in the neuron by current injected through the recording microelectrode, nor were spontaneous synaptic effects observed in the neuronal cell body or myocytes (not shown). The agents were then removed by perfusion for about $40 \mathrm{~min}$ with drugfree solution. The effect of the first suprathreshold stimulus to the solitary neuron is shown in Figure 13a; an action potential (presumably the first in the neuron since it was dissociated from the ganglion 6 weeks earlier) was followed by a second, synaptically evoked action potential and then a substantial (now subthreshold) autaptic EPSP. Powerful cholinergic synapses had formed on the previously anesthetized and blocked neuron. The neuron produced a marked, atropine-sensitive hyperpolarization of the myocytes ( $b$ and $c$ ); in atropine two trains of impulses produced no detectable effcct on the spontaneously beating myocytes. Within the sensitivity of the assay, the neuron was purely cholinergic. Of the six neurons tested in this way, three were adrenergic/cholinergic, one was adrenergic/cholinergic/apparently purinergic (an atropine-resistant hyperpolarization was present), and two, for which myocyte assays were not made, evoked autaptic EPSPs and were thus at least cholinergic. Evidently, the inactivity imposed by TTX and hexamethonium does not block formation of effective junctions or (assuming adrenergic status at the outset) transmitter plasticity, at least in neonate-derived neurons. There is at least a superficial consistency between this result and the effect of high- $\mathrm{K}^{+}$medium: TTX and hexamethonium, which prevent depolarization, permit the transition and formation of cholinergic junctions; high$\mathrm{K}^{+}$medium tends to suppress expression of cholinergic junctions

(Fig. 9c: also see Walicke et al., 1977). It is known that anesthetics and synaptic blockers do not prevent formation of effective synapses by other types of neurons in culture and in vivo (for references and discussion, see Cohen et al., 1984; Crain et al., 1968; Crain and Peterson, 1974).

\section{Discussion}

\section{The adrenergic-to-cholinergic transition in culture}

In considering these findings, it should be remembered that the superior cervical ganglion, from which the cultured principal neurons were derived, innervates a variety of target tissues, including several glands, smooth muscles (e.g., vascular, irideal), brown fat, certain parasympathetic neurons, and probably the heart. The dissociated principal neurons were plated at random on the islands of cardiac myocytes. Because it seems unlikely that the microculture environment selected rigorously for neurons that innervated the heart, we assume that most of the microcultured neurons innervated other target cells in vivo. Thus, the unconventional synaptic functions described here may be associated, in development or adult life, with the sympathetic innervation of tissues other than the heart. For example, Landis (1983) and Landis and Keefe (1983) report evidence for an adrenergic-to-cholinergic transition, apparently via dual status, during development of the sympathetic innervation of eccrine sweat glands, a transition not known (or plausible) in the sympathetic innervation of the heart. A puzzle, not yet resolved, is the induction of pronounced cholinergic function in sympathetic neurons by cultured cardiac cells, in spite of the fact that adult cardiac myocytes receive an adrenergic sympathetic innervation in vivo. Fukada (1980) raised the possibility that this cholinergic induction is suppressed during sympathetic development in vivo by the high neonatal concentration of plasma corticosterone. It is imaginable that denervated cardiac cells in culture release the CM factor (in the presence of adult serum with a lower corticosterone content) as part of an early developmental program directed at the cholinergic parasympathetic cardiac ganglion, which forms in the heart before the onset of sympathetic function (for references and discussion, see, for example, De Champlain et al., 1970; Mills, 1978; Owman et al., 1971; Standen, 1977; Taylor, 1977).

\section{Are all the neonate-derived neurons plastic and does the transition always occur via dual status?}

The high proportion of the singly assayed neonate-derived neurons that displayed at-least-cholinergic function after the first week in culture (Fig. $9 a$; about $78 \%$ of the total in this graph), the high incidence of at-least-adrencrgic/cholincrgic function (about $66 \%$ of the neurons appropriately assayed) and direct evidence for the transition in 14 serially assayed neurons (Fig. 4) leave no doubt that a large majority of the microcultured neonate-derived neurons can initiate a transition, like previously studied neurons in mass cultures, and do so via dual status, although most neurons did not complete the transition under the microculture conditions used. However, it is possible that some of the microcultured neurons were unable to undergo the transition, as some apparently purely adrenergic, neonate-derived neurons were encountered after many weeks in culture (e.g., the 80-d-old $\mathrm{N}_{2}$ of Fig. 8). Under the conditions we studied, if transitions can occur via a null state, only a small minority of microcultured neurons (cf. the null-null neuron of Fig. 4a) display this behavior. We do not know why the neurons of Figure $4 a$ showed no visible change in status, but it is possible (as all but the null-null neuron were dual in function at first assay) that the neurons were undergoing the transition at a rate too slow to be detected (most of the assay intervals were rather short). 
Can a neuron gain a new transmitter function and lose an old one?

Previous biochemical, morphological, and electrophysiological work on mass cultures (reviewed by Bunge et al., 1978; O'Lague et al., 1978c; Patterson, 1978) demonstrated a large change in transmitter status but left open the possibility that the transition is from predominantly adrenergic to predominantly cholinergic (in the notation of Fig. $4, \mathrm{~A}^{+} / \mathrm{C} \rightarrow \mathrm{A} / \mathrm{C}^{+}$, not $\mathrm{A} \rightarrow \mathrm{C}$ ). To investigate the status of individual neurons at rather advanced culture ages (4-7 weeks), Reichardt and Patterson (1977) biochemically assayed single neurons grown in a somewhat different microculture environment than that described here and found that almost all neurons detectably synthesized only one of the two transmitters; in retrospect, however, it appears that the biochemical assays were insufficiently sensitive to detect dual status reliably (rather old microcultures and correspondingly large neurons were studied because the limited sensitivity of the biochemical assays was recognized). Higgins et al. (1981) and Iacovitti et al. (1981) reported immunoreactivity for catecholamine-synthesizing enzymes in cultured sympathetic neurons that possessed cholinergic functions; these neurons were grown for up to 7 weeks under rather different conditions from those described here.

Any conclusion about the acquisition of new transmitter functions or the loss of old ones is limited by the sensitivity of the assay. However, because of the hyperinnervation of a compact group of target cells that respond electrically to both transmitters, the microculture procedure offers an especially stringent test of the physiologically significant state(s) of a neuron. In Figures 4, 5,6, and 12, we report neurons that apparently lacked either adrenergic or cholinergic function; in the previous paper we also reported singly assayed neurons that appeared to be monofunctional (Furshpan et al., 1986a). These observations thus provide clear evidence for qualitative changes in the functionally significant transmitter status of the neurons (e.g., the reduction of adrenergic function to physiologically undetectable levels). One reservation, however, about the apparent absence of cholinergic or adrenergic function at the outset or end of the transition is the possibility of transmitter actions that do not change the membrane potential or beating rate of the myocytes. Another reservation is that other target cells (perhaps cells that the neuron would have innervated in vivo) might be more sensitive to the transmitter(s) of interest than are the cardiac myocytes.

At least some of the neurons that appeared to be purely cholinergic in the myocyte assay retained adrenergic properties. For example, in the experiment shown in Figure 6, after the neuron had become apparently purely cholinergic, a small proportion of its vesicles had granular cores following incubation of the culture in 5-hydroxydopamine (Fig. 7); a similar effect was observed in the cholinergic sympathetic innervation of the adult sweat gland (Landis and Keefe, 1983). It also seems likely that some of the apparently purely cholinergic neurons observed in microculturc rclcased catccholamines in amounts below the detection sensitivity of the assay. Do such residual properties imply the gratuitous expression of adrenergic properties without physiological significance? The possibility of gratuitous synaptic properties is an increasing concern to cytochemists and biochemists (e.g., Björklund et al., 1985; Grzanna and Coyle, 1978; Hökfelt et al., 1984; Jaeger et al., 1984; Landis et al., 1983; Ross et al., 1984) and reinforces interest in sensitive assays of synaptic function.

Adrenergic/cholinergic plasticity in developing populations of cells derived from the neural crest in birds has been extensively investigated by LeDouarin and colleagues (for a review, see LeDouarin, 1980), but it is not yet known whether this occurs in a single, plastic cell type or is the result of selection between two unalterable cell types. Plasticity of transmitter status has recently been reported in several types of developing mammalian neurons in culture and in vivo (reviewed by Black et al., 1984; see also Björklund et al., 1985; Kessler, 1984; Sawchenko and Swanson, 1985). These cases involve at least large changes in expression of adrenergic, cholinergic, or peptidergic properties. The most extensive evidence for a transmitter switch in vivo has been obtained for the developing sympathetic innervation of the eccrine sweat glands by Landis (1983) and Landis and Keefe (1983).

\section{Variability in the rate of the transition}

Landis (1980) provided strong fine-structural evidence that in mass cultures the transition occurs more rapidly in neurons grown in the presence of CM than in neurons grown in several other "less cholinergic" conditions. The microculture assays, both single and serial, provide additional evidence for variable transition rates and also evidence consistent with the idea that in microcultures the rate can be effectively zero (i.e., under the particular conditions the neuron did not undergo the transition)-for example, the neurons of Figure $9 a$ that did not acquire detectable cholinergic function after many weeks (neuron $\mathrm{N}_{2}$ of Fig. 8 is one of these). The variability in the rate or ability to undergo the transition may depend on "intrinsic" differences between the neurons, differcnces in the microculture environment, or both. Intrinsic differences might be induced in embryonic neurons in connection with the identity of their eventual target cells. Differences in the environment might arise, even for two neurons in the same microculture, through contact of ccll bodics with different types of cardiac cells or if rapidly growing neurites of one neuron altered the behavior of cardiac cells toward later-arriving neurites of another neuron. Another variable might be the size of the neuritic tree (e.g., its volume, number of junctions) at the onset of the transition.

\section{Are adult neurons plastic?}

Our limited observations suggest that most adult-derived neurons are less responsive to the "cholinergic" microculture environment than most neonate-derived neurons (see also Wakshull et al., 1979a, b). This is consistent with the report of Patterson and Chun (1977b) that the responsiveness of masscultured neonate-derived neurons to $\mathrm{CM}$ falls progressively to a low level during the first 7 weeks in culture and with the finding of R. Nishi (unpublished observations) that mass cultures of adult-derived neurons synthesize and store less $\mathrm{ACh}$, in response to $\mathrm{CM}$, than do mass cultures of neonate-derived neurons. The serial-assay experiment illustrated in Figure 12 is our strongest evidence for plasticity in adult-derived neurons. The apparent sensitivity to $\mathrm{CM}$ in a group of neurons from 1-year-old rats (see Results) is also consistent with plasticity. If it is assumed that adrenergic/cholinergic dual status does not occur in adult neurons in vivo (but see below), the presence of this status in cultured adult neurons is by itself a demonstration of plasticity.

Several cases of large changes in the content of transmitter or its enzymes in a population of adult neurons have been reported. There are examples in the digestive and reproductive systems (e.g., Adham and Schenk, 1969; Alm et al., 1979; Sjöberg et al., 1977; Sjöstrand and Swedin, 1976; Thorbert et al., 1979; Tucek et al., 1976). Katz et al. (1983) reported a sharp decline in tyrosine hydroxylase activity and immunoreactivity in petrosal sensory neurons after axotomy; apparently it is not yet known whether this is the result of altered axonal transport [cf. the rapid loss of NE, AChE, and MAO from cell bodies of axotomized rat sympathetic neurons with accumulation of these substances at the site of injury (Eränkö and Härkönen, 1965; Härkönen, 1964)], the result of a change in enzyme metabolism, or both. Adler and Black (1984) recently reported a marked increase in the substance $P$ content of explants of the superior cervical ganglion of the adult rat cultured for periods up to a 
week. Sawchenko and Swanson (1985) report marked alteration in the content of two peptides, corticotropin releasing factor and vasopressin, in certain paraventricular neurons after adrenalectomy. Björklund et al. (1985) report immunoreactivity for tyrosine hydroxylase and neuron peptide $\mathrm{Y}$ in axons, presumably parasympathetic, in the rat iris after sympathectomy.

\section{Multiple-transmitter status}

When dual status was discovered with the microculture procedure (Furshpan et al., 1976; Landis, 1976), it was less conventional than it now seems, given much recent evidence for coexistence of putative transmitters or transmitter enzymes, evidence that began with the demonstration of somatostatin-like immunoreactivity in sympathetic principal neurons of the rat and guinea pig (Hökfelt et al., 1977; for reviews, see, for example, Cuello, 1982; Hökfelt et al., 1980a, 1984; Lundberg and Hökfelt, 1983), and a growing number of cases in which there is strong evidence for dual function in vivo, for example, amine/ peptide function (see Campbell et al., 1982; Jan and Jan, 1983; Lundberg, 1981) and amine/purine function (for references, see Sneddon and Westfall, 1984). The microculture procedure offers advantages for investigation of multiple-transmitter status in vertebrate neurons, as the procedure circumvents the problems of interpretation that arise because of neuronal heterogeneity in intact nerves or ganglia, the possibility of false-positives or falsenegatives in immunocytochemistry (e.g., Hökfelt et al., 1984), and the possibility, discussed above, of gratuitous expression of a synaptic property without obvious function.

We have considered elsewhere (Potter ct al., 1981a) the origin of the popular version of "Dale's Principle" (a neuron secretes only one transmitter) and concluded that this was never a legitimate principle (see also Hökfelt et al., 1980; Paton, 1982). The authentic version of "Dale's Principle" [a neuron secretes the same transmitter(s) from all its endings] is clearly a useful idea, but we know of no convincing example or counterexample in vertebrate or invertebrate neurons. The finding that the endings and varicosities of microcultured solitary neurons have a rather uniform fine structure that corresponds to their physiological status (Fig. 3) is consistent with this idea. It would be of interest to show that individual varicosities of a dual-transmitter neuron are similar in function to the summed function of the entire axonal arbor.

\section{Adrenergic/cholinergic dual status in adult sympathetic neurons in vivo: The Burn-and-Rand hypothesis}

Adrenergic/cholinergic status in adult sympathetic principal neurons in vivo was proposed by Burn and Rand (1959, 1965) as an explanation for a variety of observations. Among these was the effect of injecting ACh into a target tissue in the presence of atropine to block the postsynaptic effect of the ACh: The response of the target tissue resembled that produced by sympathetic activity. It was proposed that the injected ACh acted via neuronal nicotinic receptors to release NE from sympathetic endings. This proposal was widely accepted, but its significance was disputed. Burn and Rand suggested that sympathetic fibers normally secrete ACh to induce their own secretion of NE (the "cholinergic-link" hypothesis), a form of adrenergic/cholinergic dual function. The microculture experiments reported here add to much previous evidence (e.g., review by Ferry, 1966) that the cholinergic-link hypothesis is not valid in its simplest version: In many microculture trials, hexamethonium always failed to prevent secretion of NE or the other transmitters, at concentrations that fully blocked nicotinic receptors at autapses in the same microcultures (e.g., Fig. $5 e_{1}$, Fig. $8 a_{2}-a_{6}$ and $b_{2,3}$ ). However, the present experiments demonstrate that adrenergic/cholinergic dual status does occur in developing and adult sympathetic principal neurons in culture. As noted above, there is strong evidence that this status occurs during development of the in- nervation of eccrine sweat glands in vivo (Landis, 1983; Landis and Keefe, 1983). The functional significance of dual status in these cases is quite different from that of the "cholinergic-link" hypothesis.

Does this status also occur in adult sympathetic neurons in vivo, as Burn and Rand proposed? In spite of much investigation, this matter is apparently still open to doubt for a variety of reasons, including the heterogeneity of sympathetic neurons (for which evidence is rapidly rising, e.g., Hökfelt et al., 1980a, b), the frequent inability to block competing synaptic effects completely in attempts to unmask a cholinergic effect, and the limited or uncertain specificity of some of the common pharmacological agents. An interesting example of these difficulties is the study of the nictitating membrane of the cat. A long series of reports (e.g., Duval et al., 1984; Langer and Pinto, 1976; Mirkin and Cervoni, 1962; Reas and Tsai, 1966) have eliminated the possibility that $\mathrm{ACh}$ plays as important a role in sympathetic control of this tissue as that suggested by Burn and Rand (1960), but the observations appear compatiblc with the idea that a minority of conventional cholinergic axons is present in the sympathetic innervation and/or that $\mathrm{ACh}$ is a minor cotransmitter in some sympathetic axons. These ideas would rationalize the otherwise unexplained observation that the normal mcmbranc is highly sensitive to $\mathrm{ACh}$ as well as to NE (e.g., Trendelenburg, 1962) and becomes supersensitive to both $\mathrm{ACh}$ and NE when denervated.

A second experimental approach to the question whether adrenergic/cholinergic status occurs in adult sympathetic neurons in vivo has been to examine the character of the synaptic vesicles at terminals in certain target tissues. Tranzer et al. (1969) preloaded such terminals with NE or 5-hydroxydopamine before fixation and reported that "virtually all" the vesicles acquired the dense cores associated with storage of catecholamines; it was concluded that all the vesicles are adrenergic in character. We know of no comparable study of the nictitating membrane of the cat. Moreover, it is not yet known whether some synaptic vesicles are dual in status, i.e., capable of storing both ACh and catecholamine. Landis and Keefe (1983) report several lines of cytochemical evidence consistent with weak adrenergic function in the cholinergic sympathetic innervation of the plantar sweat glands in adult rats. We are impressed by the apparently smoothly graded character and plasticity of adrenergic and cholinergic states in cultured neurons and urge caution in interpreting existing evidence for the conventional view that all adult sympathetic neurons in vivo express these states in a strictly exclusive manner.

While (at least) adrenergic/cholinergic status was displayed by about two-thirds of the neonate-derived neurons that were appropriately assayed and some adult-derived neurons, it was not the most complicated status encountered, as can be seen in Figures 5 and 8 . Purinergic and NAE functions are described in the papers by Furshpan et al. (1986b) and Matsumoto et al. (in press), where the complexity of the transmitter repertoire is further discussed.

\section{References}

Adham, N., and E. A. Schenk (1969) Autonomic innervation of the rat vagina, cervix and uterus and its cyclic variation. Am. J. Obstet. Gynecol. 104: 508-516.

Adler, J. E., and I. B. Black (1984) Plasticity of Substance P in mature and aged sympathetic neurons in culture. Science 225: 1499-1500.

Alm, P., A. Björklund, C. Owman, and G. Thorbert (1979) Tyrosine hydroxylase and dopa decarboxylase activities in the guinea-pig uterus: Further evidence for functional adrenergic denervation in association with pregnancy. Neuroscience 4: 145-154.

Björklund, H., T. Hökfelt, M. Goldstein, L. Terenius, and L. Olson (1985) Appearance of the noradrenergic markers tyrosine hydroxylase and neuropeptide $\mathrm{Y}$ in cholinergic nerves of the iris following sympathectomy. J. Neurosci. 5: 1633-1643. 
Black, I. B., J. E. Adler, C. F. Dreyfus, G. M. Jonakait, D. M. Katz, E. F. La Gamma, and K. M. Markey (1984) Neurotransmitter plasticity at the molecular level. Science 225: 1266-1270.

Burn, J. H., and M. J. Rand (1959) Sympathetic postganglionic mechanism. Nature 184: 163-165.

Burn, J. H., and M. J. Rand (1960) Sympathetic postganglionic cholinergic fibres. Br. J. Pharmacol. 15: 56-66.

Burn, J. H., and M. J. Rand (1965) Acetylcholine in adrenergic transmission. Annu. Rev. Pharmacol. 5: 163-182.

Bunge, R., M. Johnson, and C. D. Ross (1978) Nature and nurture in development of the autonomic neuron. Science 199: 1409-1416.

Campbell, G., I. L. Gibbons, J. L. Morris, J. B. Furness, M. Costa, J. R. Oliver, A. M. Beardsley, and R. Murphy (1982) Somatostatin is contained in and released from cholinergic nerves in the heart of the toad Bufo marinus. Neuroscience 7: 2013-2023.

Cohen, M. W., M. Greschner, and M. Tucci (1984) In vivo development of cholinesterase at a neuromuscular junction in the absence of motor activity in Xenopus laevis. J. Physiol. (Lond.) 348: 57-66.

Crain, S. M., and E. R. Peterson (1974) Development of neural connections in culture. Ann. NY Acad. Sci. 228: 6-B4.

Crain, S. M., M. B. Bornstein, and E. R. Peterson (1968) Maturation of cultured embryonic CNS tissues during chronic exposure to agents which prevent bioelectric activity. Brain Res. 8: 363-372.

Cuello, A. C., ed. (1982) Co-Transmission, MacMillan, London.

De Champlain, J., L. Olson, T. Malmfors, and C. Sachs (1970) Fluorescent morphology of the developing peripheral adrenergic nerves in the rat. Acta Physiol. Scand. 80: 276-288.

Duval, N., P. E. Hicks, and S. Z. Langer (1984) Reserpine-resistant reponses to nerve stimulation in the cat nictitating membrane are due to newly synthesized transmitter. Br. J. Pharmacol. 83: 406P.

Eränkö, O., and M. Härkönen (1965) Effect of axon division on the distribution of noradrenaline and acetylcholinesterase in sympathetic neurons of the rat. Acta Physiol. Scand. 63: 411-412.

Ferry, C. B. (1966) Cholinergic link hypothesis in adrenergic neuroeffector transmission. Physiol. Rev. 46: 420-456.

Fukada, K. (1980) Hormonal control of neurotransmitter choice in sympathetic neuron cultures. Nature 287: 553-555.

Furshpan, E. J., P. R. MacLeish, P. H. O'Lague, and D. D. Potter (1976) Chemical transmission between rat sympathetic neurons and cardiac myocytes developing in microcultures: Evidence for cholinergic, adrenergic and dual-function neurons. Proc. Natl. Acad. Sci. USA 73: $4225-4229$.

Furshpan, E. J., D. D. Potter, and S. C. Landis (1982) On the transmitter repertoire of sympathetic neurons in culture. Harvey Lect. 76 : 149-191.

Furshpan, E. J., S. C. Landis, S. G. Matsumoto, and D. D. Potter (1986a) Synaptic functions in rat sympathetic neurons in microcultures. I. Secretion of norepinephrine and acetylcholine. J. Neurosci. 6: 1061-1079.

Furshpan, E. J., D. D. Potter, and S. G. Matsumoto (1986b) Synaptic functions in rat sympathetic neurons in microcultures. III. A purinergic effect on cardiac myocytes. J. Neurosci. 6: 1099-1107.

Grzanna, R., and J. T. Coyle (1978) Dopamine- $\beta$-hydroxylase in rat submandibular ganglion cells which lack norepinephrine. Brain Res. 151: 206-214.

Härkönen, M. (1964) Carboxylic esterases, oxidative enzymes and catecholamines in the superior cervical ganglion of the rat and the effect of pre- and postganglionic nerve division. Acta Physiol. Scand. (Suppl. 237) 63: 1-94.

Higgins, D. I. Iacovitti, T. H. Joh, and H. Burton (1981) The immunocytochemical localization of tyrosine hydroxylase within rat sympathetic neurons that release acetylcholine in culture. J. Neurosci. 1: $126-131$.

Hökfelt, T. (1968) In vitro studies on central and peripheral monoamine neurons at the ultrastructural level. Z. Zellforsch. 91: 1-74.

Hökfelt, T., L. G. Elfin, R. Elde, M. Schultzberg, M. Goldstein, and R. Luft (1977) Occurrence of somatostatin-like immunoreactivity in some peripheral sympathetic noradrenergic neurons. Proc. Natl. Acad. Sci. USA 74: 3587-3591.

Hökfelt, T., O. Johansson, A. Ljungdahl, J. M. Lundberg, and M. Schultzberg (1980a) Peptidergic neurones. Nature 284: 515-521.

Hökfelt, T., J. M. Lundberg, M. Schultzberg, O. Johansson, A. Ljungdahl, and J. Rehfeld (1980b) Coexistence of peptides and putative transmitters in neurons. In Neural Peptides and Neuronal Communication, E. Costa and M. Trabucchi, eds., pp. 1-23, Raven, New York.
Hökfelt, T., O. Johansson, and M. Goldstein (1984) Chemical anatomy of the brain. Science 225: 1326-1334.

Iacovitti, L., T. H. Joh, D. H. Park, and R. P. Bunge (1981) Dual expression of neurotransmitter synthesis in cultured autonomic neurons. J. Neurosci. 1: 685-690.

Jacobowitz, D., and G. B. Koelle (1965) Histochemical correlations of acetylcholinesterase and catecholamines in postganglionic autonomic nerves of the cat, rabbit and guinea pig. J. Pharmacol. Exp. Ther. 148: 225-237.

Jaeger, C. B., D. A. Ruggiero, V. R. Albert, D. H. Park, T. H. Joh, and D. J. Reis (1984) Aromatic L-amino acid decarboxylase in the rat brain: Immunocytochemical localization in neurons of the brain stem. Neuroscience 11: 691-713.

Jan, Y. N., and L. Y. Jan (1983) Coexistence and corelease of cholinergic and peptidergic transmitters in frog sympathetic ganglia. Fed. Proc. 42: 2929-2933.

Johnson, M. (1978) Adult rat dissociated sympathetic neurons in culture: Morphological and cytochemical studies. Soc. Neurosci. Abstr. 4: (\#343).

Johnson, M., D. Ross, M. Meyers, R. Rees, R. Bunge, E. Wakshull, and H. Burton (1976) Synaptic vesicle cytochemistry changes when cultured sympathetic neurons develop cholinergic interactions. Nature 262: 308-310.

Johnson, M. I., C. D. Ross, M. Meyers, E. L. Spitznagel, and R. P. Bunge (1980) Morphological and biochemical studies on the development of cholinergic properties in cultured sympathetic neurons. I. Correlative changes in choline acetyltransferase and synaptic vesicle cytochemistry. J. Cell Biol. 84: 680-691.

Katz, D. M., K. A. Markey, M. Goldstein, and I. B. Black (1983) Expression of catecholaminergic characteristics by primary sensory neurons in the normal adult rat in vivo. Proc. Natl. Acad. Sci. USA 80: 3526-3531.

Kessler, J. A. (1984) Environmental regulation of peptide neurotransmitter phenotypic expression. Soc. Neurosci. Abstr. 10: 8.1

Landis, S. C. (1976) Rat sympathetic ncurons and cardiac myocytes developing in microcultures: Correlation of the fine structure of endings with neurotransmitter function in single neurons. Proc. Natl. Acad. Sci. USA 73: 4220-4224.

Landis, S. C. (1978) Growth cones of cultured sympathetic neurons contain adrenergic vesicles. J. Cell Biol. 78: R8-R14.

Landis, S. C. (1980) Developmental changes in neurotransmitter properties of dissociated sympathetic neurons: A cytochemical study of the effects of medium. Dev. Biol. 77: 349-361.

Landis, S. C. (1983) Development of cholinergic sympathetic neurons: Evidence for neurotransmitter plasticity in vivo. Fed. Proc. 42: 16331638.

Landis, S. C., and D. Keefe (1983) Evidence for neurotransmitter plasticity in vivo: Developmental changes in properties of cholinergic sympathetic neurons. Dev. Biol. 98: 349-372.

Landis, S. C., P. R. MacLeish, D. D. Potter, E. J. Furshpan, and P. H. Patterson (1976) Synapses formed between dissociated sympathetic neurons: The influence of conditioned medium. Soc. Neurosci. Abstr. 6: $197(\# 280)$.

Landis, S. C., P. C. Jackson, and J. R. Fredieu (1983) Catecholaminergic properties of neurons and preganglionic axons in the rat ciliary ganglion. Soc. Neurosci. Abstr. 9: 937.

Langer, S. Z., and J. E. B. Pinto (1976) Possible involvement of a transmitter different from norepinephrine in the residual responses to nerve stimulation of the cat nictitating membrane after pretreatment with reserpine. J. Pharmacol. Exp. Ther. 196: 697-713.

LeDouarin, N. M. (1980) The ontogeny of the neural crest in avian embryo chimaeras. Nature 286: 663-669.

Lundberg, J. M. (1981) Evidence for coexistence of vasoactive intestinal polypeptide (VIP) and acetylcholine in neurons of cat exocrine glands. Morphological, biochemical and functional studies. Acta Physiol. Scand. (Suppl.) 496: 1-57.

Lundberg, J. M., and T. Hökfelt (1983) Coexistence of peptides and classical neurotransmitters. Trends Neurosci. 325-333.

MacLeish, P. R. (1976) Synapse formation in cultures of dissociated rat sympathetic neurons grown on dissociated rat heart cells, $\mathrm{Ph} . \mathrm{D}$. thesis, Harvard University.

Mains, R. E., and P. H. Patterson (1973) Primary cultures of dissociated sympathetic neurons. I. Establishment of long-term growth in culture and studies of differentiated properties. J. Cell Biol. 59: 329345.

Matsumoto, S. G., D. Sah, D. D. Potter, and E. J. Furshpan (in press) 
Synaptic functions in rat sympathetic neurons in microcultures. IV A slow, excitatory effect on cardiac myocytes and the variety of multiple transmitter states. J. Neurosci.

Mills, E. (1978) Time course for development of vagal inhibition of the heart in neonatal rats. Life Sci. 23: 2717-2720.

Mirkin, B. L., and P. Cervoni (1962) The adrenergic nature of neurohumoral transmission in the cat nictitating membrane following treatment with reserpine. J. Pharmacol. Exp. Ther. 138: 301-308.

O'Lague, P. H., P. R. MacLeish, C. A. Nurse, P. Claude, E. J. Furshpan, and D. D. Potter (1976) Physiological and morphological studies on developing sympathetic neurons in dissociated cell culture. Cold Spring Harbor Symp. Quant. Biol. 40: 399-407.

O'Lague, P. H., D. D. Potter, and E. J. Furshpan (1978a) Studies on rat sympathetic neurons developing in cell culture. I. Growth characteristics and electrophysiological properties. Dev. Biol. 67: 384403.

O'Lague, P. H., E. J. Furshpan, and D. D. Potter (1978b) Studies on rat sympathetic neurons developing in cell culture. II. Synaptic mechanisms. Dev. Biol. 67: 404-423.

O'Lague, P. H., D. D. Potter, and E. J. Furshpan (1978c) Studies on rat sympathetic neurons developing in cell culture. III. Cholinergic transmission. Dev. Biol. 67: 424-443.

Owman, C., N.-O. Sjöberg, and G. Swedin (1971) Histochemical and chemical studies on pre- and post-natal development of the different systcms of 'short' and 'long' adrencrgic ncurons in periphcral organs of the rat. Z. Zellforsch. 116: 319-341.

Paton, W. D. M. (1982) Foreword. In Co-Transmission, A. C. Cuello, ed., pp. VII-IX, MacMillan, London.

Patterson, P. H. (1978) Environmental determination of autonomic neurotransmitter functions. Annu. Rev. Neurosci. 1: 1-17.

Patterson, P. H., and L. L. Y. Chun (1974) The influence of nonneuronal cells on catecholamine and acetylcholine synthesis and accumulation in cultures of dissociated sympathetic neurons. Proc. Natl. Acad. Sci. USA 71: 3607-3610.

Patterson, P. H., and L. L. Y. Chun (1977a) Induction of acetylcholine synthesis in primary cultures of dissociated rat sympathetic neurons. I. Effects of conditioned medium. Dev. Biol. 56: 263-280.

Patterson, P. H., and L. L. Y. Chun (1977b) The induction of acetylcholine synthesis in primary cultures of dissociated rat sympathetic neurons. II. Developmental aspects. Dev. Biol. 60: 473-481.

Potter, D. D., S. C. Landis, and E. J. Furshpan (1980) Dual function during development of rat sympathetic neurones in culture. J. Exp. Biol. 89: 57-71

Potter, D. D., E. J. Furshpan, and S. C. Landis (1981a) Multipletransmitter status and "Dale's Principle." Neurosci. Comment. I: $1-9$.

Potter, D. D., S. C. Landis, and E. J. Furshpan (1981b) Adrenergiccholinergic dual function in cultured sympathetic neurons of the rat. Ciba Found. Symp. 83: 139-150.

Potter, D. D., S. C. Landis, and E. J. Furshpan (1981c) Chemical differentiation of sympathetic neurons. In Neurosecretion and Brain Peptides, J. B. Martin, S. Reichlin, and K. L. Bick, eds., pp. 275-285, Raven, New York.

Potter, D. D., E. J. Furshpan, and S. C. Landis (1983) Transmitter status in cultured rat sympathetic neurons: Plasticity and multiple function. Fed. Proc. 42: 1626-1632.
Reas, H. W., and T. H. Tsai (1966) The antagonism by atropine of the response of the nictitating membrane to sympathetic nerve stimulation. J. Pharmacol. Exp. Ther. 152: 186-196.

Reichardt, L. F., and P. H. Patterson (1977) Neurotransmitter synthesis and uptake by isolated sympathetic neurones in microcultures. Nature 270: 147-151.

Ross, C. A., D. A. Ruggiero, M. P. Meeley, D. H. Park, T. H. Joh, and D. J. Reis (1984) A new group of neurons in hypothalamus containing phenylethanolamine N-methyltransferase (NMT) but not tyrosine hydroxylase. Brain Res. 306: 349-353.

Sawchenko, P. E., and L. W. Swanson (1985) Localization, colocalization and plasticity of corticotropin-releasing factor immunoreactivity in rat brain. Fed. Proc. 44: 221-227.

Sjöberg, N.-O., E. D. B. Johansson, C. Owman, E. Rosengren, and B. Walles (1977) Cyclic fluctuation in noradrenaline transmitter of the monkey oviduct. Acta Obstet. Gynecol. Scand. 56: 139-143.

Sjöstrand, N. O., and G. Swedin (1976) Influences of age, growth, castration and testosterone treatment on the noradrenaline levels of the ductus deferens and the auxiliary male reproductive glands of the rat. Acta Physiol. Scand. 98: 323-338.

Snedden, P., and D. P. Westfall (1984) Pharmacological evidence that adenosine triphosphate and noradrenaline are co-transmitters in the guinea-pig vas deferens. J. Physiol. (Lond.) 347: 561-580.

Standen, N. B. (1977) Postnatal development of inotropic responses to nerve stimulation and tyramine in rat atria. Pfluegers Arch. 370: 99-101.

Taylor, I. M. (1977) The development of innervation in the rat atrioventricular node. Cell Tissue Res. 178: 73-82.

Thorbert, G., P. Alm, A. B. Björklund, C. Owman, and N.-O. Sjöberg (1979) Adrenergic innervation of the human uterus. Disappearance of the transmitter and transmitter-forming enzymes during pregnancy. Am. J. Obstet. Gynecol. 135: 223-226.

Tranzer, I. P., H. Thoenen, R. L. Snipes, and J. G. Richards (1969) Recent developments on the ultrastructural aspect of adrenergic nerve endings in various experimental conditions. Prog. Brain Res. 31: 3346.

Trendelenburg, U. (1962) The action of acetylcholine on the nictitating membrane of the spinal cat. J. Pharmacol. Exp. Ther. 135: 39-44.

Tuĉek, S., D. Kostirova, and E. Gutmann (1976) Testosterone-induced changes of choline acetyltransferase and cholinesterase activities in rat levater ani muscle. J. Neurol. Sci. 27: 353-362.

Wakshull, E., M. I. Johnson, and H. Burton (1979a) Postnatal sympathetic neurons in culture. I. A comparison with embryonic neurons. J. Neurophysiol. 42: 1410-1425.

Wakshull, E., M. I. Johnson, and H. Burton (1979b) Postnatal rat sympathetic neurons in culture. II. Synaptic transmission by postnatal neurons. J. Neurophysiol. 42: 1426-1436.

Walicke, P. A., R. B. Campenot, and P. H. Patterson (1977) Determination of transmitter function by neuronal activity. Proc. Natl. Acad. Sci. USA 74: 5767-5771.

Wolinsky, E., and P. H. Patterson (1983) Tyrosine hydroxylase activity decreases with induction of cholinergic properties in cultured sympathetic neurons. J. Neurosci. 3: 1495-1500.

Wolinsky, E., and P. H. Patterson (1985) Rat serum contains a developmentally regulated cholinergic inducing activity. J. Neurosci. 5: 1509-1512. 